\title{
Vanishing viscosity limit for the compressible Navier-Stokes system via measure-valued solutions
}

\author{
Danica Basarić@
}

\begin{abstract}
We identify a class of measure-valued solutions of the barotropic Euler system on a general (unbounded) spatial domain as a vanishing viscosity limit for the compressible Navier-Stokes system. Then we establish the weak (measure-valued)-strong uniqueness principle, and, as a corollary, we obtain strong convergence to the Euler system on the lifespan of the strong solution.
\end{abstract}

Mathematics Subject Classification. 35Q30, 35Q31, 76N10.

Keywords. Measure-valued solution of the Euler system, Vanishing viscosity limit for the compressible Navier-Stokes system, Weak (measure-valued)-strong uniqueness principle, General (unbounded) domain.

\section{Introduction}

We consider the compressible Euler system with damping

$$
\begin{aligned}
& \partial_{t} \varrho+\operatorname{div}_{x} \mathbf{m}=0 \\
& \partial_{t} \mathbf{m}+\operatorname{div}_{x}\left(\frac{\mathbf{m} \otimes \mathbf{m}}{\varrho}\right)+\nabla_{x} p(\varrho)+a \mathbf{m}=0
\end{aligned}
$$

here $\varrho=\varrho(t, x)$ denotes the density, $\mathbf{m}=\mathbf{m}(t, x)$ the momentum - with the convection that the convective term is equal to zero whenever $\varrho=0$ - and $p=p(\varrho)$ the pressure. The term $a \mathbf{m}$, with $a \geq 0$, represents "friction". We will study the system on the set $(t, x) \in(0, T) \times \Omega$, where $T>0$ is a fixed time, $\Omega \subseteq \mathbb{R}^{N}$ with $N=2,3$, can be a bounded or unbounded domain, along with the boundary condition

$$
\left.\mathbf{m} \cdot \mathbf{n}\right|_{\partial \Omega}=0
$$


for all $t \in[0, T]$; if $\Omega$ is unbounded, we impose the condition at infinity

$$
\varrho \rightarrow \bar{\varrho}, \quad \mathbf{m} \rightarrow 0 \quad \text { as }|x| \rightarrow \infty,
$$

with a constant $\bar{\varrho} \geq 0$. We also consider the following initial data

$$
\varrho(0, \cdot)=\varrho_{0}, \quad \mathbf{m}(0, \cdot)=\mathbf{m}_{0},
$$

with $\varrho_{0} \geq 0$. We finally assume that the pressure $p$ is given by the isentropic state equation

$$
p(\varrho)=A \varrho^{\gamma},
$$

where $\gamma>1$ is the adiabatic exponent and $A>0$ is a constant.

Our goal is to identify a class of generalized - dissipative measure valued $(D M V)$ solutions - for the Euler system (1), (2) as a vanishing viscosity limit of the Navier-Stokes equations. More specifically, we start considering the set

$$
\Omega_{R}=\Omega \cap B_{R}, \quad B_{R}=\left\{x \in \mathbb{R}^{N}:|x|<R\right\},
$$

where we assume $\Omega_{R}$ to be at least a Lipschitz domain, and we consider the Navier-Stokes system:

$$
\begin{aligned}
& \partial_{t} \varrho_{R}+\operatorname{div}_{x}\left(\varrho_{R} \mathbf{u}_{R}\right)=0, \\
& \partial_{t}\left(\varrho_{R} \mathbf{u}_{R}\right)+\operatorname{div}_{x}\left(\varrho_{R} \mathbf{u}_{R} \otimes \mathbf{u}_{R}\right)+\nabla_{x} p\left(\varrho_{R}\right)=\frac{1}{R} \operatorname{div}_{x} \mathbb{S}\left(\nabla_{x} \mathbf{u}_{R}\right)-a \varrho_{R} \mathbf{u}_{R} ;
\end{aligned}
$$

now $\mathbf{u}_{R}=\mathbf{u}_{R}(t, x)$ is the velocity and $\mathbb{S}_{R}=\mathbb{S}\left(\nabla_{x} \mathbf{u}_{R}\right)$ is the viscous stress, which we assume to be a linear function of the velocity gradient, more specifically to satisfy the Newton's rheological law

$$
\mathbb{S}_{R}=\mathbb{S}\left(\nabla_{x} \mathbf{u}_{R}\right)=\mu\left(\nabla_{x} \mathbf{u}_{R}+\nabla_{x}^{T} \mathbf{u}_{R}-\frac{2}{N}\left(\operatorname{div}_{x} \mathbf{u}_{R}\right) \mathbb{I}\right)+\eta\left(\operatorname{div}_{x} \mathbf{u}_{R}\right) \mathbb{I}
$$

where $\mu>0, \eta \geq 0$ are constants. Introducing $\lambda=\eta-\frac{2}{N} \mu$ we also have

$$
\mathbb{S}\left(\nabla_{x} \mathbf{u}_{R}\right)=\mu\left(\nabla_{x} \mathbf{u}_{R}+\nabla_{x}^{T} \mathbf{u}_{R}\right)+\lambda\left(\operatorname{div}_{x} \mathbf{u}_{R}\right) \mathbb{I}, \quad \mu>0, \lambda \geq-\frac{2}{N} \mu .
$$

As our goal is to perform the vanishing viscosity limit for the NavierStokes system, we impose the complete slip boundary conditions on $\partial \Omega$ :

$$
\left.\mathbf{u}_{R} \cdot \mathbf{n}\right|_{\partial \Omega}=0,\left(\mathbb{S}_{R} \cdot \mathbf{n}\right) \times\left.\mathbf{n}\right|_{\partial \Omega}=0,
$$

and the no-slip boundary conditions on $\partial B_{R}$ :

$$
\left.\mathbf{u}_{R}\right|_{\partial B_{R}}=0,
$$

for all $t \in[0, T]$. Conditions (11) and (12) may be compatible but they do not give rise to any extra analytical problem assuming that $\partial B_{R} \cap \partial \Omega=\emptyset$ for $R$ large enough, meaning that $\partial \Omega$ is a compact set. That is $\Omega$ is either (i) bounded, or (ii) exterior domain, or (iii) $\Omega=\mathbb{R}^{N}$. For the sake of simplicity, we restrict ourselves to these three cases.

Finally, we impose the initial conditions independent of $R$ :

$$
\varrho_{R}(0, \cdot)=\varrho_{0}, \quad\left(\varrho_{R} \mathbf{u}_{R}\right)(0, \cdot)=\mathbf{m}_{0} \quad \text { in } \Omega_{R},
$$

where $\varrho_{0}, \mathbf{m}_{0}$ are the initial conditions of the Euler system as in (5). 
The strategy of identifying the vanishing viscosity limit with a measurevalued solution to the Euler system can be seen as the "compressible" counterpart of the pioneering work from Di Perna and Majda [4] in the incompressible case. The compressible case was treated by Sueur [18] on a bounded domain by means of the relative energy method. Our goal is to propose an alternative approach based on the concept of dissipative measure-valued solutions and extend the result to a more general class of domains. A similar limit problem was also considered by Březina and Mácha [2] on the flat torus, where the starting system is the compressible Navier-Stokes one with some extra terms modelling non-local interaction forces, and by Chen and Glimm [3] on the whole domain $\mathbb{R}^{3}$, with a Kolmogorov-type hypothesis. Notice that the concept of dissipative measure-valued solution is of independent interest and has been used recently in the analysis of convergence of certain numerical schemes, see $[9]$.

The paper is organized as follows. In Sect. 2 we show that the solutions of the Navier-Stokes system converge to the measure-valued solution of the Euler system with damping in the zero viscosity limit, cf. Theorem 2.3. Sect. 3 is devoted to the proof of the weak-strong uniqueness principle for the Euler system with damping, i.e., if the system admits a solution in the classical sense then it must coincide with the measure-valued solution emanating from the same initial data, cf. Theorem 3.3. Despite of the standard Euler system, the linear damping term guarantees the existence of a strong solution and therefore the result can be applied to concrete examples, cf. $[15,16]$ in the case of a bounded domain, and $[12,13,17]$ for the whole domain. Finally, combining the two main results of the previous sections, we can conclude in Sect. 4 that solutions of the Navier-Stokes system converge to smooth solution of the Euler system as long as the latter exists, cf. Theorem 4.1 .

\section{From the Navier-Stokes to the Euler system}

\subsection{Weak formulation}

Choosing a constant background density $\bar{\varrho} \geq 0$, the Navier-Stokes system (7), (8) can be rewritten as

$$
\begin{aligned}
& \partial_{t}\left(\varrho_{R}-\bar{\varrho}\right)+\operatorname{div}_{x}\left(\varrho_{R} \mathbf{u}_{R}\right)=0, \\
& \partial_{t}\left(\varrho_{R} \mathbf{u}_{R}\right)+\operatorname{div}_{x}\left(\varrho_{R} \mathbf{u}_{R} \otimes \mathbf{u}_{R}\right)+\nabla_{x}\left[p\left(\varrho_{R}\right)-p(\bar{\varrho})\right] \\
& \quad=\frac{1}{R} \operatorname{div}_{x} \mathbb{S}\left(\nabla_{x} \mathbf{u}_{R}\right)-a \varrho_{R} \mathbf{u}_{R} .
\end{aligned}
$$

If we multiply both equations (7), (8) by test functions and integrate over the domain $\Omega_{R}$, knowing that the densities $\varrho_{R}$ and the momenta $\varrho_{R} \mathbf{u}_{R}$ are weakly continuous in time, we get the weak formulation of our problem:

$$
\left[\int_{\Omega_{R}}\left(\varrho_{R}-\bar{\varrho}\right) \varphi(t, \cdot) d x\right]_{t=0}^{t=\tau}=\int_{0}^{\tau} \int_{\Omega_{R}}\left[\left(\varrho_{R}-\bar{\varrho}\right) \partial_{t} \varphi+\varrho_{R} \mathbf{u}_{R} \cdot \nabla_{x} \varphi\right] d x d t
$$


for any $\tau \in[0, T)$ and all $\varphi \in C_{c}^{1}\left([0, T] \times \bar{\Omega}_{R}\right)$, and

$$
\begin{aligned}
{\left[\int_{\Omega_{R}} \varrho_{R} \mathbf{u}_{R} \cdot \boldsymbol{\varphi}(t, \cdot) d x\right]_{t=0}^{t=\tau}=} & \int_{0}^{\tau} \int_{\Omega_{R}}\left(\varrho_{R} \mathbf{u}_{R} \cdot \partial_{t} \boldsymbol{\varphi}+\varrho_{R} \mathbf{u}_{R} \otimes \mathbf{u}_{R}: \nabla_{x} \boldsymbol{\varphi}\right) d x d t \\
& +\int_{0}^{\tau} \int_{\Omega_{R}}\left[p\left(\varrho_{R}\right)-p(\bar{\varrho})\right] \operatorname{div}_{x} \boldsymbol{\varphi} d x d t \\
& -\int_{0}^{\tau} \int_{\Omega_{R}}\left[\frac{1}{R} \mathbb{S}\left(\nabla_{x} \mathbf{u}_{R}\right): \nabla_{x} \boldsymbol{\varphi}+a \varrho_{R} \mathbf{u}_{R} \cdot \boldsymbol{\varphi}\right] d x d t,
\end{aligned}
$$

for any $\tau \in[0, T)$ and all $\varphi \in C_{c}^{1}\left([0, T] \times \bar{\Omega} \cap B_{R} ; \mathbb{R}^{N}\right)$ with $\left.\varphi \cdot \mathbf{n}\right|_{\partial \Omega}=0$.

Multiplying (8) by $\mathbf{u}$ and introducing the pressure potential $P$ as the solution of the equation

$$
\varrho P^{\prime}(\varrho)-P(\varrho)=p(\varrho),
$$

which, for instance, in our case can be taken as

$$
P(\varrho)=\varrho \int_{\bar{\varrho}}^{\varrho} \frac{p(z)}{z^{2}} d z
$$

(notice in particular that $P(\bar{\varrho})=0$ ), we get the energy equality

$$
\begin{aligned}
& \frac{d}{d t} \int_{\Omega_{R}}\left[\frac{1}{2} \varrho_{R}\left|\mathbf{u}_{R}\right|^{2}+P\left(\varrho_{R}\right)\right] d x+a \int_{\Omega_{R}} \varrho_{R}\left|\mathbf{u}_{R}\right|^{2} d x \\
& \quad+\frac{1}{R} \int_{\Omega_{R}} \mathbb{S}\left(\nabla_{x} \mathbf{u}_{R}\right): \nabla_{x} \mathbf{u}_{R} d x=0 .
\end{aligned}
$$

Integrating the first equation over $\Omega_{R}$ along with conditions (11), (12), we get

$$
\frac{d}{d t} \int_{\Omega_{R}}\left(\varrho_{R}-\bar{\varrho}\right) d x=0 \Rightarrow \frac{d}{d t} \int_{\Omega_{R}} P^{\prime}(\bar{\varrho})\left(\varrho_{R}-\bar{\varrho}\right) d x=0 .
$$

Since $P(\bar{\varrho})=0$, we can rewrite $(18)$ as

$$
\begin{aligned}
& \frac{d}{d t} \int_{\Omega_{R}}\left[\frac{1}{2} \varrho_{R}\left|\mathbf{u}_{R}\right|^{2}+P\left(\varrho_{R}\right)-P^{\prime}(\bar{\varrho})\left(\varrho_{R}-\bar{\varrho}\right)-P(\bar{\varrho})\right] d x \\
& \quad+a \int_{\Omega_{R}} \varrho_{R}\left|\mathbf{u}_{R}\right|^{2} d x+\frac{1}{R} \int_{\Omega_{R}} \mathbb{S}\left(\nabla_{x} \mathbf{u}_{R}\right): \nabla_{x} \mathbf{u}_{R} d x=0
\end{aligned}
$$

from which the energy inequality follows

$$
\begin{gathered}
\int_{\Omega_{R}}\left[\frac{1}{2} \varrho_{R}\left|\mathbf{u}_{R}\right|^{2}+P\left(\varrho_{R}\right)-P^{\prime}(\bar{\varrho})\left(\varrho_{R}-\bar{\varrho}\right)-P(\bar{\varrho})\right](\tau, \cdot) d x \\
\quad+a \int_{0}^{\tau} \int_{\Omega_{R}} \varrho_{R}\left|\mathbf{u}_{R}\right|^{2} d x d t+\frac{1}{R} \int_{0}^{\tau} \int_{\Omega_{R}} \mathbb{S}\left(\nabla_{x} \mathbf{u}_{R}\right): \nabla_{x} \mathbf{u}_{R} d x d t \\
\leq \int_{\Omega_{R}}\left[\frac{1}{2} \frac{\left|\mathbf{m}_{0}\right|^{2}}{\varrho_{0}}+P\left(\varrho_{0}\right)-P^{\prime}(\bar{\varrho})\left(\varrho_{0}-\bar{\varrho}\right)-P(\bar{\varrho})\right] d x,
\end{gathered}
$$

for a.e. $\tau \in[0, T]$. For more details see [7], Section 4.2. 


\subsection{Existence of Weak Solutions}

To guarantee the existence of weak solutions, we can now use the following result.

Theorem 2.1. Let $\Omega_{R} \subset \mathbb{R}^{N}$ be a Lipschitz domain with compact boundary $\Omega_{R}=\Omega \cap B_{R}, \partial \Omega \cap \partial B_{R}=\emptyset$, and let $T>0$ be arbitrary. Suppose that the initial data satisfy

$$
\varrho_{0} \in L^{\gamma}\left(\Omega_{R}\right), \quad \varrho_{0} \geq 0 \text { a.e. in } \Omega_{R}, \quad \frac{\left|(\varrho \boldsymbol{u})_{0}\right|^{2}}{\varrho_{0}} \in L^{1}\left(\Omega_{R}\right) .
$$

Let the pressure p satisfy (6) with

$$
\gamma>\frac{N}{2}
$$

Then the Navier-Stokes system (7)-(13) admits a weak solution $\left[\varrho_{R}, \boldsymbol{u}_{R}\right]$ in $(0, T) \times \Omega_{R}$ such that

1. the density $\varrho_{R}=\varrho_{R}(t, x)$ is a non-negative function a.e. in $(0, T) \times \Omega_{R}$ and satisfies

$$
\varrho_{R} \in C_{\text {weak }}\left([0, T] ; L^{\gamma}\left(\Omega_{R}\right)\right)
$$

the velocity $\boldsymbol{u}_{R}=\boldsymbol{u}_{R}(t, x)$ satisfies

$$
\boldsymbol{u}_{R} \in L^{2}\left(0, T ; W^{1,2}\left(\Omega_{R} ; \mathbb{R}^{N}\right)\right),\left.\boldsymbol{u}_{R} \cdot \boldsymbol{n}\right|_{\partial \Omega}=0,\left.\boldsymbol{u}_{R}\right|_{\partial B_{R}}=0
$$

the momentum $\varrho_{R} \boldsymbol{u}_{R}=\left(\varrho_{R} \boldsymbol{u}_{R}\right)(t, x)$ satisfies

$$
\varrho_{R} \boldsymbol{u}_{R} \in C_{\text {weak }}\left([0, T] ; L^{\frac{2 \gamma}{\gamma+1}}\left(\Omega_{R} ; \mathbb{R}^{N}\right)\right) ;
$$

2. the weak formulations of the continuity equation (16) and of the momentum balance (17) are satisfied in $(0, T) \times \Omega_{R}$;

3. the energy inequality (19) holds for a.e. $\tau \in[0, T]$.

The proof follows the same line as in [6], Theorem 7.1. The fact that the boundary conditions are different on $\partial \Omega$ and $\partial B_{R}$ does not present any extra difficulty as the closures of these two components of the boundary are disjoint.

\subsection{Limit passage}

Starting from the family $\left\{\varrho_{R}-\bar{\varrho}, \mathbf{m}_{R}=\varrho_{R} \mathbf{u}_{R}\right\}_{R>0}$ of dissipative weak solutions to the reformulated Navier-Stokes system (14), (15) with the same initial data (13), and extending $\mathbf{u}_{R}$ to be zero and $\varrho_{R}$ as $\varrho$ outside $B_{R}$, we can now replace $\Omega_{R}$ by $\Omega$ in the previous integrals (16), (17) and (19); more precisely, from now on we will consider

$$
\left[\int_{\Omega}\left(\varrho_{R}-\bar{\varrho}\right) \varphi(t, \cdot) d x\right]_{t=0}^{t=\tau}=\int_{0}^{\tau} \int_{\Omega}\left[\left(\varrho_{R}-\bar{\varrho}\right) \partial_{t} \varphi+\varrho_{R} \mathbf{u}_{R} \cdot \nabla_{x} \varphi\right] d x d t
$$


for any $\tau \in[0, T)$ and all $\varphi \in C_{c}^{1}([0, T] \times \bar{\Omega})$,

$$
\begin{aligned}
& {\left[\int_{\Omega} \varrho_{R} \mathbf{u}_{R} \cdot \boldsymbol{\varphi}(t, \cdot) d x\right]_{t=0}^{t=\tau}} \\
& \quad=\int_{0}^{\tau} \int_{\Omega}\left[\varrho_{R} \mathbf{u}_{R} \cdot \partial_{t} \boldsymbol{\varphi}+\varrho_{R} \mathbf{u}_{R} \otimes \mathbf{u}_{R}: \nabla_{x} \boldsymbol{\varphi}+\left(p\left(\varrho_{R}\right)-p(\bar{\varrho})\right) \operatorname{div}_{x} \boldsymbol{\varphi}\right] d x d t \\
& \quad-\int_{0}^{\tau} \int_{\Omega}\left[\frac{1}{R} \mathbb{S}\left(\nabla_{x} \mathbf{u}_{R}\right): \nabla_{x} \boldsymbol{\varphi}+a \varrho_{R} \mathbf{u}_{R} \cdot \boldsymbol{\varphi}\right] d x d t
\end{aligned}
$$

for any $\tau \in[0, T)$ and all $\varphi \in C_{c}^{1}\left([0, T] \times \bar{\Omega} ; \mathbb{R}^{N}\right),\left.\boldsymbol{\varphi} \cdot \mathbf{n}\right|_{\partial \Omega}=0$, and

$$
\begin{aligned}
\int_{\Omega} & {\left[\frac{1}{2} \varrho_{R}\left|\mathbf{u}_{R}\right|^{2}+P\left(\varrho_{R}\right)-P^{\prime}(\bar{\varrho})\left(\varrho_{R}-\bar{\varrho}\right)-P(\bar{\varrho})\right](\tau, \cdot) d x } \\
& +a \int_{0}^{\tau} \int_{\Omega} \varrho_{R}\left|\mathbf{u}_{R}\right|^{2} d x d t+\frac{1}{R} \int_{0}^{\tau} \int_{\Omega_{R}} \mathbb{S}\left(\nabla_{x} \mathbf{u}_{R}\right): \nabla_{x} \mathbf{u}_{R} d x d t \\
& \leq \int_{\Omega}\left[\frac{1}{2} \frac{\left|\mathbf{m}_{0}\right|^{2}}{\varrho_{0}}+P\left(\varrho_{0}\right)-P^{\prime}(\bar{\varrho})\left(\varrho_{0}-\bar{\varrho}\right)-P(\bar{\varrho})\right] d x,
\end{aligned}
$$

for a.e. $\tau \in[0, T]$. Note that this is correct for $R$ large enough as the test functions are compactly supported in $\Omega_{R}$.

Now, we suppose that the initial data have been chosen on $\Omega$ in such a way that the initial energy is finite:

$$
\int_{\Omega}\left[\frac{1}{2} \frac{\left|\mathbf{m}_{0}\right|^{2}}{\varrho_{0}}+P\left(\varrho_{0}\right)-P^{\prime}(\bar{\varrho})\left(\varrho_{0}-\bar{\varrho}\right)-P(\bar{\varrho})\right] d x \leq E_{0} .
$$

We then easily deduce from the energy inequality (22) that

$$
\begin{aligned}
& \operatorname{ess} \sup _{t \in(0, T)}\left\|\sqrt{\varrho_{R}} \mathbf{u}_{R}(t, \cdot)\right\|_{L^{2}\left(\Omega ; \mathbb{R}^{N}\right)} \leq c\left(E_{0}\right), \\
& \operatorname{ess} \sup \left\|\left(P\left(\varrho_{R}\right)-P^{\prime}(\bar{\varrho})\left(\varrho_{R}-\bar{\varrho}\right)-P(\bar{\varrho})\right)(t, \cdot)\right\|_{L^{1}(\Omega)} \leq c\left(E_{0}\right), \\
& t \in(0, T) \\
& \frac{1}{R} \int_{0}^{T} \int_{\Omega} \mathbb{S}\left(\nabla_{x} \mathbf{u}_{R}\right): \nabla_{x} \mathbf{u}_{R} d x d t \leq c\left(E_{0}\right),
\end{aligned}
$$

where the bounds are independent of $R$. Next, from (26), we can deduce that

$$
\frac{1}{R} \int_{0}^{T}\left\|\mathbb{S}\left(\nabla_{x} \mathbf{u}_{R}\right)(t, \cdot)\right\|_{L^{2}\left(\Omega ; \mathbb{R}^{N} \times \mathbb{R}^{N}\right)}^{2} d t \leq c\left(E_{0}\right) .
$$

We can now use the relation

$$
P(\varrho)-P^{\prime}(\bar{\varrho})(\varrho-\bar{\varrho})-P(\bar{\varrho}) \geq c(\bar{\varrho}) \begin{cases}(\varrho-\bar{\varrho})^{2} & \text { for } \frac{\bar{\varrho}}{2}<\varrho<2 \bar{\varrho} \\ \left(1+\varrho^{\gamma}\right) & \text { otherwise },\end{cases}
$$

with a positive constant $c(\bar{\varrho})$ (see [8]). More precisely, following [10], Section 4.7 , we introduce the decomposition of an integrable function $h_{R}$ :

$$
h_{R}=\left[h_{R}\right]_{\text {ess }}+\left[h_{R}\right]_{\text {res }},
$$

where

$$
\left[h_{R}\right]_{e s s}=\chi\left(\varrho_{R}\right) h_{R}, \quad\left[h_{R}\right]_{r e s}=\left(1-\chi\left(\varrho_{R}\right)\right) h_{R},
$$




$$
\chi \in C_{c}^{\infty}(0, \infty), 0 \leq \chi \leq 1, \chi(r)=1 \text { for } r \in\left[\frac{\bar{\varrho}}{2}, 2 \bar{\varrho}\right] .
$$

Then we have

$$
\begin{aligned}
& \underset{t \in(0, T)}{\operatorname{ess} \sup }\left\|\left[\varrho_{R}-\bar{\varrho}\right]_{e s s}(t, \cdot)\right\|_{L^{2}(\Omega)} \\
& \quad=\underset{t \in(0, T)}{\operatorname{ess} \sup _{\Omega}} \int_{\Omega}\left(\varrho_{R}-\bar{\varrho}\right)^{2} \chi\left(\varrho_{R}\right)(t, \cdot) d x \\
& \quad \leq \frac{1}{c(\bar{\varrho})} \operatorname{ess} \sup _{t \in(0, T)}\left\|\left(P\left(\varrho_{R}\right)-P^{\prime}(\bar{\varrho})\left(\varrho_{R}-\bar{\varrho}\right)-P(\varrho)\right)(t, \cdot)\right\|_{L^{1}(\Omega)} \\
& \quad \leq c\left(E_{0}\right)
\end{aligned}
$$

and

$$
\begin{aligned}
& \operatorname{ess} \sup \left\|\left[\varrho_{R}-\bar{\varrho}\right]_{r e s}(t, \cdot)\right\|_{L^{\gamma}(\Omega)} \\
& t \in(0, T) \\
& =\operatorname{ess}_{t \in(0, T)} \int_{\Omega}\left|\varrho_{R}-\bar{\varrho}\right|^{\gamma}\left(1-\chi\left(\varrho_{R}\right)\right)(t, \cdot) d x \\
& \lesssim \operatorname{ess}_{t \in(0, T)} \int_{\Omega}\left(1+\varrho^{\gamma}\right)\left(1-\chi\left(\varrho_{R}\right)\right)(t, \cdot) d x \\
& \leq \frac{1}{c(\bar{\varrho})} \operatorname{ess}_{t \in(0, T)}\left\|\left(P\left(\varrho_{R}\right)-P^{\prime}(\bar{\varrho})\left(\varrho_{R}-\bar{\varrho}\right)-P(\bar{\varrho})\right)(t, \cdot)\right\|_{L^{1}(\Omega)} \\
& \leq c\left(E_{0}\right) \text {, }
\end{aligned}
$$

where $\lesssim$ means modulo a multiplication constant. In particular this implies that

$$
\begin{aligned}
& {\left[\varrho_{R}-\bar{\varrho}\right]_{e s s} \stackrel{*}{\rightarrow} f_{\varrho-\bar{\varrho}} \quad \text { in } L^{\infty}\left(0, T ; L^{2}(\Omega)\right),} \\
& {\left[\varrho_{R}-\bar{\varrho}\right]_{r e s} \stackrel{*}{\rightarrow} g_{\varrho-\bar{\varrho}} \quad \text { in } L^{\infty}\left(0, T ; L^{\gamma}(\Omega)\right) ;}
\end{aligned}
$$

passing to suitable subsequences as the case may be; defining $\varrho-\bar{\varrho}:=f_{\varrho-\bar{\varrho}}+g_{\varrho-\bar{\varrho}}$, we have that

$$
\varrho_{R}-\bar{\varrho} \stackrel{*}{\rightarrow} \varrho-\bar{\varrho} \quad \text { in } L^{\infty}\left(0, T ; L^{2}+L^{\gamma}(\Omega)\right) .
$$

We can repeat the same procedure for the momenta; indeed, using (24) we obtain

$$
\begin{aligned}
\operatorname{ess~sup}_{t \in(0, T)}\left\|\left[\varrho_{R} \mathbf{u}_{R}\right]_{e s s}(t, \cdot)\right\|_{L^{2}(\Omega)} & =\operatorname{ess}_{t \in(0, T)} \int_{\Omega} \varrho_{R} \cdot \varrho_{R}\left|\mathbf{u}_{R}\right|^{2} \chi\left(\varrho_{R}\right)(t, \cdot) d x \\
& \leq 2 \varrho \operatorname{ess~sup}_{t \in(0, T)}\left\|\sqrt{\varrho_{R}} \mathbf{u}_{R}(t, \cdot)\right\|_{L^{2}(\Omega)} \\
& \leq c\left(E_{0}\right)
\end{aligned}
$$

we also have

$$
\begin{aligned}
\operatorname{ess~sup}_{t \in(0, T)}\left\|\left[\sqrt{\varrho_{R}}\right]_{r e s}(t, \cdot)\right\|_{L^{2 \gamma}(\Omega)} & =\operatorname{essips}_{t \in(0, T)} \int_{\Omega} \varrho_{R}^{\gamma}\left(1-\chi\left(\varrho_{R}\right)\right)(t, \cdot) d x \\
& \leq \operatorname{essips}_{t \in(0, T)} \int_{\Omega}\left(\varrho_{R}^{\gamma}+1\right)\left(1-\chi\left(\varrho_{R}\right)\right)(t, \cdot) d x
\end{aligned}
$$




$$
\leq c\left(E_{0}\right),
$$

which, together with (24) and Hölder's inequality with $p=\gamma+1$, gives

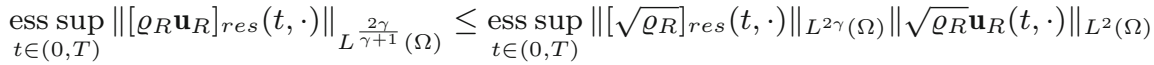

$$
\begin{aligned}
& \leq c\left(E_{0}\right) \text {. }
\end{aligned}
$$

Then we obtain

$$
\varrho_{R} \mathbf{u}_{R} \stackrel{*}{\rightarrow} \mathbf{m} \quad \text { in } L^{\infty}\left(0, T ; L^{2}+L^{\frac{2 \gamma}{\gamma+1}}(\Omega)\right),
$$

passing to suitable subsequences as the case may be. In a similar way we have

$$
\begin{aligned}
\operatorname{ess~sup}_{t \in(0, T)}\left\|\left[p\left(\varrho_{R}\right)-p(\bar{\varrho})\right]_{\text {ess }}(t, \cdot)\right\|_{L^{2}(\Omega)} & =\underset{t \in(0, T)}{\operatorname{ess} \sup _{\Omega}} \int_{\Omega}\left|p\left(\varrho_{R}\right)-p(\bar{\varrho})\right|^{2} \chi\left(\varrho_{R}\right)(t, \cdot) d x \\
& \leq p^{\prime}(2 \bar{\varrho}) \operatorname{ess} \sup _{t \in(0, T)}\left\|\left[\varrho_{R}-\bar{\varrho}\right]_{e s s}(t, \cdot)\right\|_{L^{2}(\Omega)} \\
& \leq c\left(E_{0}\right),
\end{aligned}
$$

and

$$
\begin{aligned}
& \underset{t \in(0, T)}{\operatorname{ess} \sup }\left\|\left[p\left(\varrho_{R}\right)-p(\bar{\varrho})\right]_{r e s}(t, \cdot)\right\|_{L^{1}(\Omega)} \\
& \quad=A \underset{t \in(0, T)}{\operatorname{ess} \sup _{\Omega}} \int_{\Omega}\left|\varrho_{R}^{\gamma}-\bar{\varrho}^{\gamma}\right|\left(1-\chi\left(\varrho_{R}\right)\right)(t, \cdot) d x \\
& \quad \leq A \max \left\{\bar{\varrho}^{\gamma}, 1\right\} \underset{t \in(0, T)}{\operatorname{ess} \sup } \int_{\Omega}\left(1+\varrho_{R}^{\gamma}\right)\left(1-\chi\left(\varrho_{R}\right)\right)(t, \cdot) d x \\
& \quad \leq c\left(E_{0}\right) .
\end{aligned}
$$

Furthermore, noticing that

$$
\left|\varrho_{R} \mathbf{u}_{R} \otimes \mathbf{u}_{R}\right| \lesssim \frac{1}{2} \varrho_{R}\left|\mathbf{u}_{R}\right|^{2},
$$

from (24) we deduce that also the convective terms are uniformly bounded in the non-reflexive space $L^{1}((0, T) \times \Omega)$, or better, in $L^{\infty}\left(0, T ; L^{1}(\Omega)\right)$.

There are two disturbing phenomena that may occur to bounded sequences in $L^{1}$ : oscillations and concentrations. The idea is then to see $L^{1}((0, T)$ $\times \Omega)$ as embedded in the space of bounded Radon measures $\mathcal{M}([0, T] \times \bar{\Omega})$ - that happens to be the dual to the separable space $C_{0}([0, T] \times \bar{\Omega})=\overline{C_{c}([0, T] \times \bar{\Omega})}\|\cdot\|_{\infty}$ - through the identification

$$
\mu_{f}(\varphi)=\int_{0}^{T} \int_{\Omega} f \varphi d x d t, \quad \text { for all } \varphi \in C_{0}([0, T] \times \bar{\Omega}),
$$

if $f \in L^{1}((0, T) \times \Omega)$.

Accordingly, we may assume

$$
\begin{aligned}
& \varrho_{R}-\bar{\varrho} \stackrel{*}{\rightarrow} \varrho-\bar{\varrho} \quad \text { in } L^{\infty}\left(0, T ; L^{2}+L^{\gamma}(\Omega)\right) ; \\
& \varrho_{R} \mathbf{u}_{R} \stackrel{*}{\rightarrow} \mathbf{m} \quad \text { in } L^{\infty}\left(0, T ; L^{2}+L^{\frac{2 \gamma}{\gamma+1}}(\Omega)\right) ; \\
& \mu_{p\left(\varrho_{R}\right)-p(\bar{\varrho})} \stackrel{*}{\rightarrow} \mu_{\{p\}-p(\bar{\varrho})} \quad \text { in } \mathcal{M}([0, T] \times \bar{\Omega}) ;
\end{aligned}
$$




$$
\begin{aligned}
& \mu_{\varrho_{R} \mathbf{u}_{R} \otimes \mathbf{u}_{R}} \stackrel{*}{\rightarrow} \mu_{\{\mathbb{M}\}} \quad \text { in } \mathcal{M}\left([0, T] \times \bar{\Omega} ; \mathbb{R}^{N} \times \mathbb{R}^{N}\right) ; \\
& \mu_{\frac{1}{2} \varrho_{R}|\mathbf{u}|^{2}+P\left(\varrho_{R}\right)-P^{\prime}(\bar{\varrho})\left(\varrho_{R}-\bar{\varrho}\right)-P(\bar{\varrho})} \stackrel{*}{\rightarrow} \mu_{\{E\}} \quad \text { in } \mathcal{M}([0, T] \times \bar{\Omega}),
\end{aligned}
$$

passing to suitable subsequences as the case may be. The notation $\mu_{\{p\}-p(\bar{\varrho})}$ means that

$$
\begin{aligned}
\left\langle\mu_{p\left(\varrho_{R}\right)-p(\bar{\varrho})} ; \varphi\right\rangle & =\int_{0}^{T} \int_{\Omega}\left[p\left(\varrho_{R}\right)-p(\bar{\varrho})\right] \varphi d x d t \rightarrow \int_{0}^{T} \int_{\Omega}[\{p\}-p(\bar{\varrho})] \varphi d x d t \\
& =\left\langle\mu_{\{p\}-p(\bar{\varrho})} ; \varphi\right\rangle
\end{aligned}
$$

as $R \rightarrow \infty$, for every $\varphi \in C_{c}([0, T] \times \bar{\Omega})$; the same holds for the other convergences.

We can now let $R \rightarrow \infty$ in the weak formulation of our initial problem (14), (15); notice that the $R$-dependent viscous stress tensor vanishes. Indeed, using (27) and Hölder's inequality we get

$$
\begin{aligned}
& \left|\frac{1}{R} \int_{0}^{T} \int_{\Omega} \mathbb{S}\left(\nabla_{x} \mathbf{u}_{R}\right): \nabla_{x} \boldsymbol{\varphi} d x d t\right| \\
& \quad \leq \frac{1}{\sqrt{R}}\left\|\frac{1}{\sqrt{R}} \mathbb{S}\left(\nabla_{x} \mathbf{u}_{R}\right)\right\|_{L^{2}((0, T) \times \Omega)}\left\|\nabla_{x} \boldsymbol{\varphi}\right\|_{L^{2}((0, T) \times \Omega)} \\
& \quad \leq \frac{c\left(E_{0}\right)}{\sqrt{R}}\left\|\nabla_{x} \boldsymbol{\varphi}\right\|_{L^{2}((0, T) \times \Omega)} .
\end{aligned}
$$

Then we get

$$
\int_{0}^{T} \int_{\Omega}\left[(\varrho-\bar{\varrho}) \partial_{t} \varphi+\mathbf{m} \cdot \nabla_{x} \varphi\right] d x d t=0
$$

for every $\varphi \in C_{c}^{1}((0, T) \times \Omega)$, and

$$
\int_{0}^{T} \int_{\Omega}\left[\mathbf{m} \cdot \partial_{t} \boldsymbol{\varphi}+\{\mathbb{M}\}: \nabla_{x} \boldsymbol{\varphi}+(\{p\}-p(\bar{\varrho})) \operatorname{div}_{x} \boldsymbol{\varphi}-a \mathbf{m} \cdot \boldsymbol{\varphi}\right] d x d t=0,
$$

for every $\varphi \in C_{c}^{1}\left((0, T) \times \Omega ; \mathbb{R}^{N}\right)$. We can equivalently write

$$
\int_{0}^{T} \int_{\Omega}\left[\varrho \partial_{t} \varphi+\mathbf{m} \cdot \nabla_{x} \varphi\right] d x d t=0
$$

for every $\varphi \in C_{c}^{1}((0, T) \times \Omega)$, and

$$
\int_{0}^{T} \int_{\Omega}\left[\mathbf{m} \cdot \partial_{t} \boldsymbol{\varphi}+\{\mathbb{M}\}: \nabla_{x} \boldsymbol{\varphi}+\{p\} \operatorname{div}_{x} \boldsymbol{\varphi}-a \mathbf{m} \cdot \boldsymbol{\varphi}\right] d x d t=0,
$$

for every $\varphi \in C_{c}^{1}\left((0, T) \times \Omega ; \mathbb{R}^{N}\right)$.

As a matter of fact, the limit for $\varrho_{R}-\bar{\varrho}$ can be strengthened to

$$
\varrho_{R}-\bar{\varrho} \rightarrow \varrho-\bar{\varrho} \text { in } C_{w e a k}\left([0, T] ; L^{2}+L^{\gamma}(\Omega)\right) ;
$$

the same holds for the limit of $\varrho_{R} \mathbf{u}_{R}$ :

$$
\varrho_{R} \mathbf{u}_{R} \rightarrow \mathbf{m} \text { in } C_{\text {weak }}\left([0, T] ; L^{2}+L^{\frac{2 \gamma}{\gamma+1}}\left(\Omega ; \mathbb{R}^{N}\right)\right) .
$$


We can then rewrite the last two integral equations as

$$
\int_{\Omega} \varrho \varphi(\tau, \cdot) d x-\int_{\Omega} \varrho \varphi(0, \cdot) d x=\int_{0}^{\tau} \int_{\Omega}\left[\varrho \partial_{t} \varphi+\mathbf{m} \cdot \nabla_{x} \varphi\right] d x d t,
$$

for any $\tau \in[0, T)$ and any $\varphi \in C_{c}^{1}([0, T] \times \bar{\Omega})$ and

$$
\begin{aligned}
\int_{\Omega} \mathbf{m} \cdot \boldsymbol{\varphi}(\tau, \cdot) d x-\int_{\Omega} \mathbf{m} \cdot \boldsymbol{\varphi}(0, \cdot) d x \\
\quad=\int_{0}^{\tau} \int_{\Omega}\left[\mathbf{m} \cdot \partial_{t} \boldsymbol{\varphi}+\{\mathbb{M}\}: \nabla_{x} \boldsymbol{\varphi}+\{p\} \operatorname{div}_{x} \boldsymbol{\varphi}-a \mathbf{m} \cdot \boldsymbol{\varphi}\right] d x d t
\end{aligned}
$$

for any $\tau \in[0, T)$ and any $\varphi \in C_{c}^{1}\left([0, T] \times \bar{\Omega} ; \mathbb{R}^{N}\right),\left.\boldsymbol{\varphi} \cdot \mathbf{n}\right|_{\partial \Omega}=0$.

Finally, using the generalization of the concept of Lebesgue point to Radon measures, we can deduce from the energy inequality (22)

$$
\int_{\Omega}\{E\}(\tau, \cdot) d x+a \int_{0}^{\tau} \int_{\Omega} \operatorname{trace}\{\mathbb{M}\} d x d t \leq \int_{\Omega}\{E\}(0, \cdot) d x,
$$

for a.e. $\tau \in(0, T)$, where

$$
\int_{\Omega}\{E\}(\tau, \cdot) d x=\lim _{\delta \rightarrow 0} \frac{1}{2 \delta} \int_{\tau-\delta}^{\tau+\delta} \int_{\Omega}\{E\} d x d t .
$$

Equations (34), (35), and (36) form a suitable platform for introducing the measure-valued solutions of the Euler system. The exact definition requires the concept of Young measure; the interested reader can find all the details in the Appendix A.

\subsection{Dissipative measure-valued solution for the compressible Euler system with damping}

Motivated by the discussion in Appendix A, we are ready to introduce the concept of dissipative measure-valued solution to the compressible Euler system with damping. It can be seen as a generalization of a similar concept introduced by Gwiazda et al. [11]. While the definition in [11] is based on the description of concentrations via the Alibert-Bouchitté defect measures [1], our approach is motivated by [5], where the mere inequality (40) is required postulating the domination of the concentrations by the energy dissipation defect. This strategy seems to fit better the studies of singular limits on general physical domains performed in the present paper.

Definition 2.2. A parametrized family of probability measures

$$
\begin{aligned}
& \nu_{t, x}:(t, x) \in(0, T) \times \Omega \rightarrow \mathcal{P}\left([0, \infty) \times \mathbb{R}^{N}\right), \\
& \nu \in L_{\text {weak }}^{\infty}\left((0, T) \times \Omega ; \mathcal{P}\left([0, \infty) \times \mathbb{R}^{N}\right)\right),
\end{aligned}
$$

is a dissipative measure-valued solution of the problem (1), (2) with the initial condition $\left\{\nu_{0, x}\right\}_{x \in \Omega}$ if 
1. the integral identity

$$
\begin{aligned}
\int_{\Omega}\left\langle\nu_{\tau, x} ; \varrho\right\rangle \varphi d x-\int_{\Omega}\left\langle\nu_{0, x} ; \varrho\right\rangle \varphi d x= & \int_{0}^{\tau} \int_{\Omega}\left[\left\langle\nu_{t, x} ; \varrho\right\rangle \partial_{t} \varphi+\left\langle\nu_{t, x} ; \mathbf{m}\right\rangle \cdot \nabla_{x} \varphi\right] d x d t \\
& +\int_{0}^{\tau} \int_{\Omega} \nabla_{x} \varphi \cdot d \mu_{c}
\end{aligned}
$$

holds for all $\tau \in[0, T)$, and for all $\varphi \in C_{c}^{1}([0, T] \times \bar{\Omega})$, where $\mu_{c} \in$ $\mathcal{M}\left([0, T] \times \bar{\Omega} ; \mathbb{R}^{N}\right)$ is a vector-valued measure;

2. the integral identity

$$
\begin{aligned}
\int_{\Omega}\left\langle\nu_{\tau, x} ; \mathbf{m}\right\rangle \cdot \boldsymbol{\varphi}(\tau, \cdot) d x-\int_{\Omega}\left\langle\nu_{0, x} ; \mathbf{m}\right\rangle \cdot \boldsymbol{\varphi}(0, \cdot) d x \\
=\int_{0}^{\tau} \int_{\Omega}\left[\left\langle\nu_{t, x} ; \mathbf{m}\right\rangle \cdot \partial_{t} \boldsymbol{\varphi}+\left\langle\nu_{t, x} ; \frac{\mathbf{m} \otimes \mathbf{m}}{\varrho}\right\rangle: \nabla_{x} \boldsymbol{\varphi}\right] d x d t \\
\quad+\int_{0}^{\tau} \int_{\Omega}\left[\left\langle\nu_{t, x} ; p(\varrho)\right\rangle \operatorname{div}_{x} \boldsymbol{\varphi}-a\left\langle\nu_{t, x} ; \mathbf{m}\right\rangle \cdot \boldsymbol{\varphi}\right] d x d t+\int_{0}^{\tau} \int_{\Omega} \nabla_{x} \boldsymbol{\varphi}: d \mu_{m}
\end{aligned}
$$

holds for all $\tau \in[0, T)$ and for all $\varphi \in C_{c}^{1}\left([0, T] \times \bar{\Omega} ; \mathbb{R}^{N}\right),\left.\boldsymbol{\varphi} \cdot \mathbf{n}\right|_{\partial \Omega}$, where $\mu_{m} \in \mathcal{M}\left([0, T] \times \bar{\Omega} ; \mathbb{R}^{N} \times \mathbb{R}^{N}\right)$ is a tensor-valued measure; both $\mu_{c}, \mu_{m}$ are called concentration measures;

3. the following inequality

$$
\begin{aligned}
\int_{\Omega} & \left\langle\nu_{\tau, x} ; \frac{1}{2} \frac{|\mathbf{m}|^{2}}{\varrho}+P(\varrho)-P^{\prime}(\bar{\varrho})(\varrho-\bar{\varrho})-P(\bar{\varrho})\right\rangle d x \\
& +a \int_{0}^{\tau} \int_{\Omega}\left\langle\nu_{t, x} ; \frac{|\mathbf{m}|^{2}}{\varrho}\right\rangle d x d t+\mathcal{D}(\tau) \\
& \leq \int_{\Omega}\left\langle\nu_{0, x} ; \frac{1}{2} \frac{|\mathbf{m}|^{2}}{\varrho}+P(\varrho)-P^{\prime}(\bar{\varrho})(\varrho-\bar{\varrho})-P(\bar{\varrho})\right\rangle d x
\end{aligned}
$$

holds for a.e. $\tau \in(0, T)$, where $\mathcal{D} \in L^{\infty}(0, T), \mathcal{D} \geq 0$ is called dissipation defect of the total energy;

4. there exists a constant $C>0$ such that

$$
\int_{0}^{\tau} \int_{\Omega} d\left|\mu_{c}\right|+\int_{0}^{\tau} \int_{\Omega} d\left|\mu_{m}\right| \leq C \int_{0}^{\tau} \mathcal{D}(t) d t
$$

for a.e. $\tau \in(0, T)$.

Now, summarizing the discussion concerning the vanishing viscosity limit of the Navier-Stokes system, we can state the first result of the present paper.

Theorem 2.3. Let $\Omega \subset \mathbb{R}^{N}, N=2,3$ be a domain with compact Lipschitz boundary and $\bar{\varrho} \geq 0$ be a given far field density if $\Omega$ is unbounded. Suppose that $\gamma>\frac{N}{2}$ and let $\varrho^{R}, \boldsymbol{u}^{R}$ be a family of weak solutions to the Navier-Stokes system $(7)-(12)$ in

$$
(0, T) \times \Omega_{R}, \Omega_{R}=\Omega \cap B_{R} .
$$

Let the corresponding initial data $\varrho_{0}, \boldsymbol{u}_{0}$ be independent of $R$ satisfying

$$
\varrho_{0} \geq 0, \quad \int_{\Omega}\left[\frac{1}{2} \varrho_{0}\left|\boldsymbol{u}_{0}\right|^{2}+P\left(\varrho_{0}\right)-P^{\prime}(\bar{\varrho})\left(\varrho_{0}-\bar{\varrho}\right)-P(\bar{\varrho})\right] d x \leq E_{0} .
$$


Then the family $\left\{\varrho^{R}, \boldsymbol{m}^{R}=\varrho^{R} \boldsymbol{u}^{R}\right\}_{R>0}$ generates, as $R \rightarrow \infty$, a Young measure $\left\{\nu_{t, x}\right\}_{t \in(0, T) ; x \in \Omega}$ which is a dissipative measure-valued solution of the Euler system with damping (1), (2).

\section{Weak-strong uniqueness}

Our next goal is to show that the dissipative measure-valued solutions introduced in the previous section satisfy an extended version of the energy inequality (39) known as relative energy inequality.

We introduce the relative energy functional:

$$
\begin{aligned}
\mathcal{E}(\nu & \left.=\nu_{t, x}(\varrho, \mathbf{m}) \mid r, \mathbf{U}\right) \\
& =\int_{\Omega}\left\langle\nu_{t, x} ; \frac{1}{2 \varrho}\left(|\mathbf{m}-\varrho \mathbf{U}|^{2}\right)+P(\varrho)-P^{\prime}(r)(\varrho-r)-P(r)\right\rangle d x
\end{aligned}
$$

If $\varrho \mapsto p(\varrho)$ is strictly increasing in $(0, \infty)$, which is true in our case, then the pressure potential $P$ is strictly convex; indeed

$$
P^{\prime \prime}(\varrho)=\frac{p^{\prime}(\varrho)}{\varrho}>0 .
$$

For a differentiable function this is equivalent in saying that the function lies above all of its tangents:

$$
P(\varrho) \geq P^{\prime}(r)(\varrho-r)+P(r)
$$

for all $\varrho, r \in(0, \infty)$, and the equality holds if and only if $\varrho=r$. Thus, we deduced that $\mathcal{E} \geq 0$, where equality holds if and only if

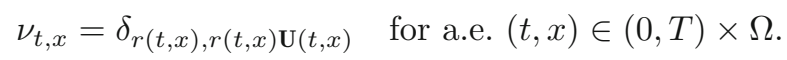

We can now prove the following

Theorem 3.1. Let $[r, \boldsymbol{U}]$ be a strong solution of the compressible Euler system with damping with compactly supported initial data so that $\boldsymbol{U} \in C_{c}^{\infty}([0, T] \times$ $\left.\bar{\Omega} ; \mathbb{R}^{N}\right)$, where in particular $\left.\boldsymbol{U} \cdot \boldsymbol{n}\right|_{\partial \Omega}=0$, and $r-\bar{\varrho} \in C_{c}^{\infty}([0, T] \times \bar{\Omega})$ with $r>0$. Let $\left\{\nu_{t, x}\right\}_{(t, x) \in(0, T) \times \Omega}$ be a dissipative measure-valued solution of the same system (in terms of the density $\varrho$ and the momentum $\boldsymbol{m}$ ), with a dissipation defect $\mathcal{D}$ and such that

$$
\nu_{0, x}=\delta_{r(0, x),(r \boldsymbol{U})(0, x)} \quad \text { for a.e. } x \in \Omega .
$$

Then $\mathcal{D}=0$ and

$$
\nu_{t, x}=\delta_{r(t, x),(r \boldsymbol{U})(t, x)} \quad \text { for a.e. }(t, x) \in(0, T) \times \Omega .
$$

Remark 3.2. Note that we must have $\bar{\varrho}>0$ if $\Omega$ is unbounded.

Proof. It is enough to prove that $\mathcal{E}(\tau)=0$ for all $\tau \in(0, T)$. We can take $\mathbf{U}$ as a test function in the momentum equation (38) to obtain 


$$
\begin{aligned}
& {\left[\int_{\Omega}\left\langle\nu_{t, x} ; \mathbf{m}\right\rangle \cdot \mathbf{U} d x\right]_{t=0}^{t=\tau}} \\
& =\int_{0}^{\tau} \int_{\Omega}\left[\left\langle\nu_{t, x} ; \mathbf{m}\right\rangle \cdot \partial_{t} \mathbf{U}+\left\langle\nu_{t, x} ; \frac{\mathbf{m} \otimes \mathbf{m}}{\varrho}\right\rangle: \nabla_{x} \mathbf{U}\right] d x d t \\
& \quad+\int_{0}^{\tau} \int_{\Omega}\left\langle\nu_{t, x} ; p(\varrho)\right\rangle \operatorname{div}_{x} \mathbf{U} d x d t-a \int_{0}^{\tau} \int_{\Omega}\left\langle\nu_{t, x} ; \mathbf{m}\right\rangle \cdot \mathbf{U} d x d t \\
& \quad+\int_{0}^{\tau} \int_{\Omega} \nabla_{x} \mathbf{U}: d \mu_{m} ;
\end{aligned}
$$

and $\frac{1}{2}|\mathbf{U}|^{2}$ as a test function in the continuity equation (37) to get

$$
\begin{aligned}
{\left[\frac{1}{2} \int_{\Omega}\left\langle\nu_{t, x} ; \varrho\right\rangle|\mathbf{U}|^{2} d x\right]_{t=0}^{t=\tau}=} & \int_{0}^{\tau} \int_{\Omega}\left[\left\langle\nu_{t, x} ; \varrho\right\rangle \mathbf{U} \cdot \partial_{t} \mathbf{U}+\left\langle\nu_{t, x} ; \mathbf{m}\right\rangle \cdot \nabla_{x} \mathbf{U} \cdot \mathbf{U}\right] d x d t \\
& +\int_{0}^{\tau} \int_{\Omega} \mathbf{U} \cdot \nabla_{x} \mathbf{U} \cdot d \mu_{c} .
\end{aligned}
$$

Finally, take $P^{\prime}(r)-P^{\prime}(\bar{\varrho})$ as test function in (37) to get

$$
\begin{aligned}
& {\left[\int_{\Omega}\left\langle\nu_{t, x} ; \varrho\right\rangle\left(P^{\prime}(r)(t, \cdot)-P^{\prime}(\bar{\varrho})\right) d x\right]_{t=0}^{t=\tau}} \\
& \quad=\int_{0}^{\tau} \int_{\Omega}\left[\left\langle\nu_{t, x} ; \varrho\right\rangle \partial_{t} P^{\prime}(r)+\left\langle\nu_{t, x} ; \mathbf{m}\right\rangle \cdot \nabla_{x} P^{\prime}(r)\right] d x d t \\
& \quad+\int_{0}^{\tau} \int_{\Omega} \nabla_{x} P^{\prime}(r) \cdot d \mu_{c} .
\end{aligned}
$$

Then, from the energy inequality (39), summing up all these terms we get

$$
\begin{aligned}
{\left[\int_{\Omega}\right.} & \left.\left\langle\nu_{t, x} ; \frac{1}{2 \varrho}|\mathbf{m}-\varrho \mathbf{U}|^{2}+P(\varrho)-\varrho P^{\prime}(r)+\bar{\varrho} P(\bar{\varrho})\right\rangle d x\right]_{t=0}^{t=\tau} \\
& +a \int_{0}^{\tau} \int_{\Omega}\left\langle\nu_{t, x} ; \frac{\mathbf{m}}{\varrho} \cdot(\mathbf{m}-\varrho \mathbf{U})\right\rangle d x d t+\mathcal{D}(\tau) \\
\leq & \int_{0}^{\tau} \int_{\Omega}\left\langle\nu_{t, x} ; \varrho \mathbf{U}-\mathbf{m}\right\rangle \cdot\left[\partial_{t} \mathbf{U}+\nabla_{x} \mathbf{U} \cdot \mathbf{U}\right] d x d t \\
& +\int_{0}^{\tau} \int_{\Omega}\left\langle\nu_{t, x} ; \frac{(\mathbf{m}-\varrho \mathbf{U}) \otimes(\varrho \mathbf{U}-\mathbf{m})}{\varrho}\right\rangle: \nabla_{x} \mathbf{U} d x d t \\
& -\int_{0}^{\tau} \int_{\Omega}\left\langle\nu_{t, x} ; p(\varrho)\right\rangle \operatorname{div} \mathbf{U}_{x} \mathbf{U} d x d t \\
& -\int_{0}^{\tau} \int_{\Omega}\left[\left\langle\nu_{t, x} ; \varrho\right\rangle \partial_{t} P^{\prime}(r)+\left\langle\nu_{t, x} ; \mathbf{m}\right\rangle \cdot \nabla_{x} P^{\prime}(r)\right] d x d t \\
& -\int_{0}^{\tau} \int_{\Omega} \nabla_{x} \mathbf{U}: d \mu_{m}+\int_{0}^{\tau} \int_{\Omega} \mathbf{U} \cdot \nabla_{x} \mathbf{U} \cdot d \mu_{c}-\int_{0}^{\tau} \int_{\Omega} \nabla_{x} P^{\prime}(r) \cdot d \mu_{c} .
\end{aligned}
$$

Notice that the term

$$
\frac{\mathbf{m}}{\varrho} \cdot(\mathbf{m}-\varrho \mathbf{U})=\frac{|\mathbf{m}|^{2}}{\varrho}-\mathbf{m} \cdot \mathbf{U}
$$


is well-defined and integrable. We have

$P(\varrho)-\varrho P^{\prime}(r)+\bar{\varrho} P(\bar{\varrho})=P(\varrho)-P^{\prime}(r)(\varrho-r)-P(r)-\left[r P^{\prime}(r)-P(r)-\bar{\varrho} P^{\prime}(\bar{\varrho})\right]$, where, since $P(\bar{\varrho})=0$,

$$
r P^{\prime}(r)-P(r)-\bar{\varrho} P^{\prime}(\bar{\varrho})=p(r)-p(\bar{\varrho}) .
$$

Then

$$
\begin{aligned}
{\left[\int_{\Omega}\left\langle\nu_{t, x} ; p(r)-p(\bar{\varrho})\right\rangle d x\right]_{t=0}^{t=\tau} } & =\int_{0}^{\tau} \int_{\Omega}\left\langle\nu_{t, x} ; \partial_{t}(p(r)-p(\bar{\varrho}))\right\rangle d x d t \\
& =\int_{0}^{\tau} \int_{\Omega}\left\langle\nu_{t, x} ; \partial_{t} p(r)\right\rangle d x d t
\end{aligned}
$$

and, using the relation $p^{\prime}(r)=r P^{\prime \prime}(r)$ along with the fact that

$$
\int_{\Omega} \operatorname{div}_{x}[p(r) \mathbf{U}] d x=\int_{\partial \Omega} p(r) \mathbf{U} \cdot \mathbf{n} d x=0,
$$

we can deduce that

$$
\begin{aligned}
\int_{\Omega}\left\langle\nu_{t, x} ; \partial_{t} p(r)\right\rangle d x & =\int_{\Omega}\left\langle\nu_{t, x} ; r \partial_{t} P^{\prime}(r)+\operatorname{div}_{x}[p(r) \mathbf{U}]\right\rangle d x \\
& =\int_{\Omega}\left\langle\nu_{t, x} ; r \partial_{t} P^{\prime}(r)+r \nabla_{x} P^{\prime}(r) \cdot \mathbf{U}+p(r) \operatorname{div}_{x} \mathbf{U}\right\rangle d x .
\end{aligned}
$$

We obtain the relative energy inequality:

$$
\begin{aligned}
{[\mathcal{E}(\nu \mid r, \mathbf{U})]_{t=0}^{t=\tau}+a \int_{0}^{\tau} \int_{\Omega}\left\langle\nu_{t, x} ; \frac{\mathbf{m}}{\varrho} \cdot(\mathbf{m}-\varrho \mathbf{U})\right\rangle d x d t+\mathcal{D}(\tau) } \\
\leq \quad \int_{0}^{\tau} \int_{\Omega}\left\langle\nu_{t, x} ; \varrho \mathbf{U}-\mathbf{m}\right\rangle \cdot\left[\partial_{t} \mathbf{U}+\nabla_{x} \mathbf{U} \cdot \mathbf{U}\right] d x d t \\
\quad+\int_{0}^{\tau} \int_{\Omega}\left\langle\nu_{t, x} ; \frac{(\mathbf{m}-\varrho \mathbf{U}) \otimes(\varrho \mathbf{U}-\mathbf{m})}{\varrho}\right\rangle: \nabla_{x} \mathbf{U} d x d t \\
\quad-\int_{0}^{\tau} \int_{\Omega}\left\langle\nu_{t, x} ; p(\varrho)-p(r)\right\rangle \operatorname{div}_{x} \mathbf{U} d x d t \\
\quad-\int_{0}^{\tau} \int_{\Omega}\left[\left\langle\nu_{t, x} ;(\varrho-r) \partial_{t} P^{\prime}(r)+(\mathbf{m}-r \mathbf{U}) \cdot \nabla_{x} P^{\prime}(r)\right\rangle d x d t\right. \\
-\int_{0}^{\tau} \int_{\Omega} \nabla_{x} \mathbf{U}: d \mu_{m}+\int_{0}^{\tau} \int_{\Omega} \mathbf{U} \cdot \nabla_{x} \mathbf{U} \cdot d \mu_{c}-\int_{0}^{\tau} \int_{\Omega} \nabla_{x} P^{\prime}(r) \cdot d \mu_{c} .
\end{aligned}
$$

Now we can use the fact that $[r, \mathbf{U}]$ is a strong solution: from the momentum equation we can deduce that

$$
\partial_{t} \mathbf{U}+\mathbf{U} \cdot \nabla_{x} \mathbf{U}=-\frac{1}{r} \nabla_{x} p(r)-a \mathbf{U}=-P^{\prime \prime}(r) \nabla_{x} r-a \mathbf{U}=-\nabla_{x} P^{\prime}(r)-a \mathbf{U}
$$

substituting, we get

$$
\begin{gathered}
{[\mathcal{E}(\nu \mid r, \mathbf{U})]_{t=0}^{t=\tau}+a \int_{0}^{\tau} \int_{\Omega}\left\langle\nu_{t, x} ; \frac{1}{2 \varrho}|\mathbf{m}-\varrho \mathbf{U}|^{2}\right\rangle d x d t+\mathcal{D}(\tau)} \\
\leq \int_{0}^{\tau} \int_{\Omega}\left\langle\nu_{t, x} ; \frac{(\mathbf{m}-\varrho \mathbf{U}) \otimes(\varrho \mathbf{U}-\mathbf{m})}{\varrho}\right\rangle: \nabla_{x} \mathbf{U} d x d t
\end{gathered}
$$




$$
\begin{aligned}
& -\int_{0}^{\tau} \int_{\Omega}\left\langle\nu_{t, x} ; p(\varrho)-p(r)\right\rangle \operatorname{div}_{x} \mathbf{U} d x d t \\
& -\int_{0}^{\tau} \int_{\Omega}\left[\left\langle\nu_{t, x} ; P^{\prime \prime}(r)(\varrho-r)\left[\partial_{t} r+\nabla_{x} r \cdot \mathbf{U}\right]\right\rangle d x d t\right. \\
& -\int_{0}^{\tau} \int_{\Omega} \nabla_{x} \mathbf{U}: d \mu_{m}+\int_{0}^{\tau} \int_{\Omega} \mathbf{U} \cdot \nabla_{x} \mathbf{U} \cdot d \mu_{c}-\int_{0}^{\tau} \int_{\Omega} \nabla_{x} P^{\prime}(r) \cdot d \mu_{c} .
\end{aligned}
$$

From the continuity equation we also have

$$
\partial_{t} r+\nabla_{x} r \cdot \mathbf{U}=-r \operatorname{div}_{x} \mathbf{U}
$$

and thus, knowing that $r P^{\prime \prime}(r)=p^{\prime}(r)$, we get

$$
\begin{aligned}
{[\mathcal{E}(\nu \mid r, \mathbf{U})]_{t=0}^{t=\tau}+a \int_{0}^{\tau} \int_{\Omega}\left\langle\nu_{t, x} ; \frac{1}{2 \varrho}|\mathbf{m}-\varrho \mathbf{U}|^{2}\right\rangle d x d t+\mathcal{D}(\tau) } \\
\leq \quad \int_{0}^{\tau} \int_{\Omega}\left\langle\nu_{t, x} ; \frac{(\mathbf{m}-\varrho \mathbf{U}) \otimes(\varrho \mathbf{U}-\mathbf{m})}{\varrho}\right\rangle: \nabla_{x} \mathbf{U} d x d t \\
\quad-\int_{0}^{\tau} \int_{\Omega}\left\langle\nu_{t, x} ; p(\varrho)-p^{\prime}(r)(\varrho-r)-p(r)\right\rangle \operatorname{div}_{x} \mathbf{U} d x d t \\
\quad-\int_{0}^{\tau} \int_{\Omega} \nabla_{x} \mathbf{U}: d \mu_{m}+\int_{0}^{\tau} \int_{\Omega} \mathbf{U} \cdot \nabla_{x} \mathbf{U} \cdot d \mu_{c}-\int_{0}^{\tau} \int_{\Omega} \nabla_{x} P^{\prime}(r) \cdot d \mu_{c} .
\end{aligned}
$$

Finally, using the fact that the initial data are the same and thus $\mathcal{E}(\nu \mid r, \mathbf{U})(0)=$ 0 , we end up to

$$
\begin{aligned}
\mathcal{E}(\nu \mid r & , \mathbf{U})(\tau)+a \int_{0}^{\tau} \int_{\Omega}\left\langle\nu_{t, x} ; \frac{1}{2 \varrho}|\mathbf{m}-\varrho \mathbf{U}|^{2}\right\rangle d x d t+\mathcal{D}(\tau) \\
\leq & \int_{0}^{\tau} \int_{\Omega}\left\langle\nu_{t, x} ;\left|\frac{(\mathbf{m}-\varrho \mathbf{U}) \otimes(\varrho \mathbf{U}-\mathbf{m})}{\varrho}\right|\right\rangle\left|\nabla_{x} \mathbf{U}\right| d x d t \\
& +\int_{0}^{\tau} \int_{\Omega}\left\langle\nu_{t, x} ;\left|p(\varrho)-p^{\prime}(r)(\varrho-r)-p(r)\right|\right\rangle\left|\operatorname{div}_{x} \mathbf{U}\right| d x d t \\
& +\int_{0}^{\tau} \int_{\Omega}\left|\nabla_{x} \mathbf{U}\right| \cdot d\left|\mu_{m}\right|+\int_{0}^{\tau} \int_{\Omega}\left|\mathbf{U} \cdot \nabla_{x} \mathbf{U}\right| \cdot d\left|\mu_{c}\right|+\int_{0}^{\tau} \int_{\Omega}\left|\nabla_{x} P^{\prime}(r)\right| \cdot d\left|\mu_{c}\right| .
\end{aligned}
$$

Since $\mathbf{U}$ and $P^{\prime}(r)-P(\bar{\varrho})$ have compact support we can control the terms $\left|\nabla_{x} \mathbf{U}\right|,\left|\operatorname{div}_{x} \mathbf{U}\right|,\left|\mathbf{U} \cdot \nabla_{x} \mathbf{U}\right|$ and $\left|\nabla_{x} P^{\prime}(r)\right|$ by some constants. It is also obvious that there exist a constant $c_{1}$ such that

$$
\left|\frac{(\mathbf{m}-\varrho \mathbf{U}) \otimes(\varrho \mathbf{U}-\mathbf{m})}{\varrho}\right| \leq \frac{c_{1}}{2 \varrho}|\mathbf{m}-\varrho \mathbf{U}|^{2},
$$

and a constant $c_{2}$ such that

$$
\left|p(\varrho)-p^{\prime}(r)(\varrho-r)-p(r)\right| \leq c_{2}\left(P(\varrho)-P^{\prime}(r)(\varrho-r)-P(r)\right) .
$$

Thus

$$
\mathcal{E}(\varrho, \mathbf{m} \mid r, \mathbf{U})(\tau)+\mathcal{D}(\tau) \leq c \int_{0}^{\tau}[\mathcal{E}(\varrho, \mathbf{m} \mid r, \mathbf{U})(t)+\mathcal{D}(t)] d t .
$$

By Gronwall lemma we obtain

$$
\mathcal{E}(\varrho, \mathbf{m} \mid r, \mathbf{U})(\tau)+\mathcal{D}(\tau) \leq 0 \quad \text { for all } \tau \in(0, T) .
$$

But since $\mathcal{E}, \mathcal{D} \geq 0$ this implies $\mathcal{D}(\tau)=0$ and $\mathcal{E}(\tau)=0$ for all $\tau \in(0, T)$. 


\subsection{Density argument}

Notice that the relative energy inequality (43) is true for general functions $r-$ $\bar{\varrho} \in C_{c}^{\infty}([0, T] \times \bar{\Omega}), \mathbf{U} \in C_{c}^{\infty}\left([0, T] \times \bar{\Omega} ; \mathbb{R}^{N}\right)$, not necessarily strong solutions to the Euler system. Then, using a density argument, we can prove the following result.

Theorem 3.3. Let $[r, \boldsymbol{U}]$ be a strong solution of the compressible Euler system with damping such that $\boldsymbol{U} \in C\left([0, T] ; H^{M}\left(\Omega ; \mathbb{R}^{N}\right)\right), M>\frac{N}{2}+1$, where in particular $\left.\boldsymbol{U} \cdot \boldsymbol{n}\right|_{\partial \Omega}=0$, and $r-\bar{\varrho} \in C\left([0, T] ; H^{M}(\Omega)\right)$ with $r>0$. Let $\left\{\nu_{t, x}\right\}_{(t, x) \in(0, T) \times \Omega}$ be a dissipative measure-valued solution of the same system (in terms of $\varrho$ and the momentum $\boldsymbol{m}$ ), with a dissipation defect $\mathcal{D}$ and such that

$$
\nu_{0, x}=\delta_{r(0, x),(r \boldsymbol{U})(0, x)} \quad \text { for a.e. } x \in \Omega \text {. }
$$

Then $\mathcal{D}=0$ and

$$
\nu_{t, x}=\delta_{r(t, x),(r \boldsymbol{U})(t, x)} \quad \text { for a.e. }(t, x) \in(0, T) \times \Omega .
$$

Proof. We will first prove that the relative energy inequality (43) holds for $[r, \mathbf{U}]$ as in our hypothesis. By density, we can find two sequences $\left\{r_{n}-\bar{\varrho}\right\}_{n \in \mathbb{N}} \subset$ $C_{c}^{\infty}([0, T] \times \bar{\Omega}),\left\{\mathbf{U}_{n}\right\}_{n \in \mathbb{N}} \subset C_{c}^{\infty}\left([0, T] \times \bar{\Omega} ; \mathbb{R}^{N}\right)$ such that

$$
\begin{aligned}
& r_{n}-\bar{\varrho} \rightarrow r-\bar{\varrho} \quad \text { in } C\left([0, T] ; H^{M}(\Omega)\right), \\
& \mathbf{U}_{n} \rightarrow \mathbf{U} \quad \text { in } C\left([0, T] ; H^{M}\left(\Omega ; \mathbb{R}^{N}\right)\right) .
\end{aligned}
$$

If we now fix $\varepsilon>0$, we know that there exists $n_{0}=n_{0}(\varepsilon)$ such that, for every $n \geq n_{0}$

$$
\begin{aligned}
& \sup _{t \in[0, T]}\left\|\left(r-r_{n}\right)(t, \cdot)\right\|_{H^{M}(\Omega)}<\varepsilon, \\
& \sup _{t \in[0, T]}\left\|\left(\mathbf{U}-\mathbf{U}_{n}\right)(t, \cdot)\right\|_{H^{M}\left(\Omega ; \mathbb{R}^{N}\right)}<\varepsilon .
\end{aligned}
$$

From now on, let $n \geq n_{0}$; for each $t \in[0, T]$ we have

$$
\begin{aligned}
\int_{\Omega}\left\langle\nu_{t, x} ; \frac{1}{2 \varrho}|\mathbf{m}-\varrho \mathbf{U}|^{2}\right\rangle d x= & \int_{\Omega}\left\langle\nu_{t, x} ; \frac{1}{2 \varrho}\left|\mathbf{m}-\varrho\left(\mathbf{U}-\mathbf{U}_{n}+\mathbf{U}_{n}\right)\right|^{2}\right\rangle d x \\
= & \int_{\Omega}\left\langle\nu_{t, x} ; \frac{1}{2 \varrho}\left|\mathbf{m}-\varrho \mathbf{U}_{n}\right|^{2}\right\rangle d x \\
& -\int_{\Omega}\left\langle\nu_{t, x} ; \mathbf{m}-(\varrho-\bar{\varrho}) \mathbf{U}_{n}\right\rangle \cdot\left(\mathbf{U}-\mathbf{U}_{n}\right)(t, \cdot) d x \\
& +\bar{\varrho} \int_{\Omega} \mathbf{U}_{n} \cdot\left(\mathbf{U}-\mathbf{U}_{n}\right)(t, \cdot) d x \\
& +\frac{1}{2} \int_{\Omega}\left\langle\nu_{t, x} ; \varrho-\bar{\varrho}\right\rangle\left|\mathbf{U}-\mathbf{U}_{n}\right|^{2}(t, \cdot) d x \\
& +\frac{\bar{\varrho}}{2} \int_{\Omega}\left|\mathbf{U}-\mathbf{U}_{n}\right|^{2}(t, \cdot) d x .
\end{aligned}
$$


Revoking notation introduced in Sect. 2.3, we focus on the last three lines: we can rewrite the first term as

$$
\begin{aligned}
& \int_{\Omega}\left\langle\nu_{t, x} ;[\mathbf{m}]_{e s s}-[\varrho-\bar{\varrho}]_{e s s} \mathbf{U}_{n}\right\rangle \cdot\left(\mathbf{U}-\mathbf{U}_{n}\right)(t, \cdot) d x \\
& \quad+\int_{\Omega}\left\langle\nu_{t, x} ;[\mathbf{m}]_{r e s}-[\varrho-\bar{\varrho}]_{r e s} \mathbf{U}_{n}\right\rangle \cdot\left(\mathbf{U}-\mathbf{U}_{n}\right)(t, \cdot) d x ;
\end{aligned}
$$

since $\left\langle\nu_{(t, \cdot)} ;[\mathbf{m}]_{e s s}-[\varrho-\bar{\varrho}]_{e s s} \mathbf{U}_{n}\right\rangle,\left(\mathbf{U}-\mathbf{U}_{n}\right)(t, \cdot) \in L^{2}\left(\Omega ; \mathbb{R}^{N}\right)$ we can apply Hölder's inequality to get

$$
\begin{aligned}
& \int_{\Omega}\left\langle\nu_{t, x} ;[\mathbf{m}]_{e s s}-[\varrho-\bar{\varrho}]_{e s s} \mathbf{U}_{n}\right\rangle \cdot\left(\mathbf{U}-\mathbf{U}_{n}\right)(t, \cdot) d x \\
& \quad \leq \sup _{t \in[0, T]}\left\|\left\langle\nu_{(t, \cdot)} ;[\mathbf{m}]_{e s s}-[\varrho-\bar{\varrho}]_{e s s} \mathbf{U}_{n}\right\rangle\right\|_{L^{2}\left(\Omega ; \mathbb{R}^{N}\right)}\left\|\left(\mathbf{U}-\mathbf{U}_{n}\right)(t, \cdot)\right\|_{L^{2}\left(\Omega ; \mathbb{R}^{N}\right)} \\
& \quad \leq C \sup _{t \in[0, T]}\left\|\left(\mathbf{U}-\mathbf{U}_{n}\right)(t, \cdot)\right\|_{H^{M}\left(\Omega ; \mathbb{R}^{N}\right)} \\
& \quad \leq C \varepsilon .
\end{aligned}
$$

We also have that $\left.\left\langle\nu_{(t, \cdot)} ;[\varrho-\bar{\varrho}]_{r e s} \mathbf{U}_{n}\right\rangle \in L^{\gamma}(K) ; \mathbb{R}^{N}\right)$ with $K$ compact and since $\gamma>\frac{2 \gamma}{\gamma+1}$ we obtain $\left\langle\nu_{(t, \cdot)} ;[\mathbf{m}]_{\text {res }}-[\varrho-\bar{\varrho}]_{\text {res }} \mathbf{U}_{n}\right\rangle \in L^{\frac{2 \gamma}{\gamma+1}}\left(\Omega ; \mathbb{R}^{N}\right)$; using the embedding of the Sobolev space into the Hölder one we get that $\left(\mathbf{U}-\mathbf{U}_{n}\right)(t, \cdot) \in$ $L^{\infty}\left(\Omega ; \mathbb{R}^{N}\right)$ and hence $\left(\mathbf{U}-\mathbf{U}_{n}\right)(t, \cdot) \in L^{p}\left(\Omega ; \mathbb{R}^{N}\right)$ for all $p \in[2, \infty]$. Since $\frac{2 \gamma}{\gamma-1}>2$, we can again apply Hölder's inequality to get

$$
\begin{aligned}
& \int_{\Omega}\left\langle\nu_{t, x} ;[\mathbf{m}]_{r e s}-[\varrho-\bar{\varrho}]_{r e s} \mathbf{U}_{n}\right\rangle \cdot\left(\mathbf{U}-\mathbf{U}_{n}\right)(t, \cdot) d x \\
& \quad \leq \sup _{t \in[0, T]}\left\|\left\langle\nu_{(t, \cdot)} ;[\mathbf{m}]_{r e s}-[\varrho-\bar{\varrho}]_{r e s} \mathbf{U}_{n}\right\rangle\right\|_{L^{\frac{2 \gamma}{\gamma+1}}\left(\Omega ; \mathbb{R}^{N}\right)}\left\|\left(\mathbf{U}-\mathbf{U}_{n}\right)(t, \cdot)\right\|_{L^{\frac{2 \gamma}{\gamma-1}}\left(\Omega ; \mathbb{R}^{N}\right)} \\
& \quad \leq C \sup _{t \in[0, T]}\left\|\left(\mathbf{U}-\mathbf{U}_{n}\right)(t, \cdot)\right\|_{H^{M}\left(\Omega ; \mathbb{R}^{N}\right)} \\
& \quad \leq C \varepsilon .
\end{aligned}
$$

For the second term we can apply Hölder's inequality:

$$
\begin{aligned}
\int_{\Omega} \mathbf{U}_{n} \cdot\left(\mathbf{U}-\mathbf{U}_{n}\right)(t, \cdot) d x & \leq \sup _{t \in[0, T]}\left\|\mathbf{U}_{n}(t, \cdot)\right\|_{L^{2}\left(\Omega ; \mathbb{R}^{N}\right)}\left\|\left(\mathbf{U}-\mathbf{U}_{n}\right)(t, \cdot)\right\|_{L^{2}\left(\Omega ; \mathbb{R}^{N}\right)} \\
& \leq C \sup _{t \in[0, T]}\left\|\left(\mathbf{U}-\mathbf{U}_{n}\right)(t, \cdot)\right\|_{H^{M}\left(\Omega ; \mathbb{R}^{N}\right)} \\
& \leq C \varepsilon .
\end{aligned}
$$

Applying the same procedure as before to the third term we get

$$
\begin{aligned}
& \int_{\Omega}\left\langle\nu_{t, x} ; \varrho-\bar{\varrho}\right\rangle\left|\mathbf{U}-\mathbf{U}_{n}\right|^{2}(t, \cdot) d x \\
& \quad=\int_{\Omega}\left\langle\nu_{t, x} ;[\varrho-\bar{\varrho}]_{e s s}+[\varrho-\bar{\varrho}]_{r e s}\right\rangle\left|\mathbf{U}-\mathbf{U}_{n}\right|^{2}(t, \cdot) d x \\
& \quad \leq \sup _{t \in[0, T]}\left\|\left\langle\nu_{(t, \cdot)} ;[\varrho-\bar{\varrho}]_{e s s}\right\rangle\right\|_{L^{2}\left(\Omega ; \mathbb{R}^{N}\right)}\left\|\left(\mathbf{U}-\mathbf{U}_{n}\right)(t, \cdot)\right\|_{L^{4}\left(\Omega ; \mathbb{R}^{N}\right)} \\
& \quad+\sup _{t \in[0, T]}\left\|\left\langle\nu_{(t, \cdot)} ;[\varrho-\bar{\varrho}]_{r e s}\right\rangle\right\|_{L^{\gamma}\left(\Omega ; \mathbb{R}^{N}\right)}\left\|\left(\mathbf{U}-\mathbf{U}_{n}\right)(t, \cdot)\right\|_{L^{\frac{2 \gamma}{\gamma-1}}\left(\Omega ; \mathbb{R}^{N}\right)} \\
& \quad \leq C \varepsilon .
\end{aligned}
$$


For the last term we simply have

$$
\int_{\Omega}\left|\mathbf{U}-\mathbf{U}_{n}\right|^{2}(t, \cdot) d x \leq \sup _{t \in[0, T]}\left\|\left(\mathbf{U}-\mathbf{U}_{n}\right)(t, \cdot)\right\|_{H^{M}\left(\Omega ; \mathbb{R}^{N}\right)}<\varepsilon .
$$

Similarly,

$$
\begin{aligned}
\int_{\Omega}\left\langle\nu_{t, x} ; P(\varrho)-P^{\prime}(r)(\varrho-r)-P(r)\right\rangle d x \\
=\int_{\Omega}\left\langle\nu_{t, x} ; P(\varrho)-P^{\prime}\left(r_{n}\right)\left(\varrho-r_{n}\right)-P\left(r_{n}\right)\right\rangle d x \\
\quad+\int_{\Omega}\left\langle\nu_{t, x} ; P^{\prime}\left(r_{n}\right)\left(\varrho-r_{n}\right)-P^{\prime}(r)(\varrho-r)\right\rangle d x \\
\quad-\int_{\Omega}\left\langle\nu_{t, x} ; P(r)-P\left(r_{n}\right)\right\rangle d x \\
=\int_{\Omega}\left\langle\nu_{t, x} ; P\left(r_{n}\right)-P^{\prime}(r)\left(r_{n}-r\right)+P(r)\right\rangle d x \\
\quad-\int_{\Omega}\left\langle\nu_{t, x} ;\left[P^{\prime}(r)-P^{\prime}\left(r_{n}\right)\right]\left(\varrho-r_{n}\right)\right\rangle d x \\
=\frac{P^{\prime \prime}\left(\xi_{1}\right)}{2} \int_{\Omega}\left(r-r_{n}\right)^{2}(t, \cdot) d x \\
\quad-P^{\prime \prime}\left(\xi_{2}\right) \int_{\Omega}\left\langle\nu_{t, x} ; \varrho-\bar{\varrho}\right\rangle\left(r-r_{n}\right) d x \\
\quad+P^{\prime \prime}\left(\xi_{2}\right) \int_{\Omega}\left(r-r_{n}\right)\left(r_{n}-\bar{\varrho}\right)(t, \cdot) d x .
\end{aligned}
$$

We can now focus on the last three lines: the first term is simply bounded as follows

$$
\int_{\Omega}\left(r-r_{n}\right)^{2}(t, \cdot) d x \leq \sup _{t \in[0, T]}\left\|\left(r-r_{n}\right)(t, \cdot)\right\|_{H^{M}(\Omega)}<\varepsilon .
$$

The second term can be rewritten as

$$
\begin{aligned}
\int_{\Omega} & \left\langle\nu_{t, x} ; \varrho-\bar{\varrho}\right\rangle\left(r-r_{n}\right)(t, \cdot) d x \\
= & \int_{\Omega}\left\langle\nu_{t, x} ;[\varrho-\bar{\varrho}]_{e s s}\right\rangle\left(r-r_{n}\right)(t, \cdot) d x \\
& \quad+\int_{\Omega}\left\langle\nu_{t, x} ;[\varrho-\bar{\varrho}]_{r e s}\right\rangle\left(r-r_{n}\right)(t, \cdot) d x \\
\leq & \sup _{t \in[0, T]}\left\|\left\langle\nu_{(t, \cdot)} ;[\varrho-\bar{\varrho}]_{e s s}\right\rangle\right\|_{L^{2}\left(\Omega ; \mathbb{R}^{N}\right)}\left\|\left(r-r_{n}\right)(t, \cdot)\right\|_{L^{2}(\Omega)} \\
& +\sup _{t \in[0, T]}\left\|\left\langle\nu_{(t, \cdot)} ;[\varrho-\bar{\varrho}]_{r e s}\right\rangle\right\|_{L^{\gamma}\left(\Omega ; \mathbb{R}^{N}\right)}\left\|\left(r-r_{n}\right)(t, \cdot)\right\|_{L^{\frac{\gamma}{\gamma-1}}(\Omega)} \\
\leq & C \varepsilon ;
\end{aligned}
$$

notice that, if $\gamma \in(1,2)$ we use the same argument as before while if $\gamma \in[2, \infty)$ we have to use the Sobolev embedding in the $L^{p}$-spaces. For the last term we 
can use Hölder inequality to get

$$
\begin{aligned}
\int_{\Omega}\left(r-r_{n}\right)\left(r_{n}-\bar{\varrho}\right)(t, \cdot) d x & \leq \sup _{t \in[0, T]}\left\|\left(r_{n}-\bar{\varrho}\right)(t, \cdot)\right\|_{L^{2}(\Omega)}\left\|\left(r-r_{n}\right)(t, \cdot)\right\|_{L^{2}(\Omega)} \\
& \leq C \sup _{t \in[0, T]}\left\|\left(r-r_{n}\right)(t, \cdot)\right\|_{H^{M}(\Omega)} \\
& \leq C \varepsilon .
\end{aligned}
$$

Repeating the same steps for each term that appears in the relative energy inequality and introducing the operator

$$
\begin{aligned}
\mathcal{L}(\nu \mid r, \mathbf{U})(\tau)= & a \int_{0}^{\tau} \int_{\Omega}\left\langle\nu_{t, x} ; \frac{\mathbf{m}}{\varrho} \cdot(\mathbf{m}-\varrho \mathbf{U})\right\rangle d x d t+\mathcal{D}(\tau) \\
& +\int_{0}^{\tau} \int_{\Omega}\left\langle\nu_{t, x} ; \mathbf{m}-\varrho \mathbf{U}\right\rangle \cdot\left[\partial_{t} \mathbf{U}+\nabla_{x} \mathbf{U} \cdot \mathbf{U}\right] d x d t \\
& -\int_{0}^{\tau} \int_{\Omega}\left\langle\nu_{t, x} ; \frac{(\mathbf{m}-\varrho \mathbf{U}) \otimes(\varrho \mathbf{U}-\mathbf{m})}{\varrho}\right\rangle: \nabla_{x} \mathbf{U} d x d t \\
& +\int_{0}^{\tau} \int_{\Omega}\left\langle\nu_{t, x} ; p(\varrho)-p(r)\right\rangle \operatorname{div}_{x} \mathbf{U} d x d t \\
& +\int_{0}^{\tau} \int_{\Omega}\left[\left\langle\nu_{t, x} ;(\varrho-r) \partial_{t} P^{\prime}(r)+(\mathbf{m}-r \mathbf{U}) \cdot \nabla_{x} P^{\prime}(r)\right\rangle d x d t\right. \\
& +\int_{0}^{\tau} \int_{\Omega} \nabla_{x} \mathbf{U}: d \mu_{m}+\int_{0}^{\tau} \int_{\Omega} \mathbf{U} \cdot \nabla_{x} \mathbf{U} \cdot d \mu_{c}-\int_{0}^{\tau} \int_{\Omega} \nabla_{x} P^{\prime}(r) \cdot d \mu_{c}
\end{aligned}
$$

we have

$$
[\mathcal{E}(\nu \mid r, \mathbf{U})(t)]_{t=0}^{t=\tau}+\mathcal{L}(\nu \mid r, \mathbf{U})(\tau) \leq\left[\mathcal{E}\left(\nu \mid r_{n}, \mathbf{U}_{n}\right)(t)\right]_{t=0}^{t=\tau}+\mathcal{L}\left(\nu \mid r_{n}, \mathbf{U}_{n}\right)(\tau)+C \varepsilon \leq C \varepsilon,
$$

for some positive constant $\mathrm{C}$, since for a test function we already proved that the relative energy inequality holds which is equivalent in saying that

$$
\left[\mathcal{E}\left(\nu \mid r_{n}, \mathbf{U}_{n}\right)(t)\right]_{t=0}^{t=\tau}+\mathcal{L}\left(\nu \mid r_{n}, \mathbf{U}_{n}\right)(\tau) \leq 0 .
$$

By the arbitrary of $\varepsilon$ we can conclude that the relative energy inequality holds for $[r, \mathbf{U}]$ as in our hypothesis.

Repeating the same passages as we did in the proof of the previous theorem, we end up to the following inequality

$$
\begin{aligned}
\mathcal{E}(\nu \mid r & , \mathbf{U})(\tau)+a \int_{0}^{\tau} \int_{\Omega}\left\langle\nu_{t, x} ; \frac{1}{2 \varrho}|\mathbf{m}-\varrho \mathbf{U}|^{2}\right\rangle d x d t+\mathcal{D}(\tau) \\
\leq & \int_{0}^{\tau} \int_{\Omega}\left\langle\nu_{t, x} ;\left|\frac{(\mathbf{m}-\varrho \mathbf{U}) \otimes(\varrho \mathbf{U}-\mathbf{m})}{\varrho}\right|\right\rangle\left|\nabla_{x} \mathbf{U}\right| d x d t \\
& +\int_{0}^{\tau} \int_{\Omega}\left\langle\nu_{t, x} ;\left|p(\varrho)-p^{\prime}(r)(\varrho-r)-p(r)\right|\right\rangle\left|\operatorname{div}_{x} \mathbf{U}\right| d x d t \\
& +\int_{0}^{\tau} \int_{\Omega}\left|\nabla_{x} \mathbf{U}\right| \cdot d\left|\mu_{m}\right|+\int_{0}^{\tau} \int_{\Omega}\left|\mathbf{U} \cdot \nabla_{x} \mathbf{U}\right| \cdot d\left|\mu_{c}\right|+\int_{0}^{\tau} \int_{\Omega}\left|\nabla_{x} P^{\prime}(r)\right| \cdot d\left|\mu_{c}\right| .
\end{aligned}
$$

The thesis now follows as before - the only thing that changes is that in this case $\mathbf{U}$ and $P(r)-P(\bar{\varrho})$ are $L^{\infty}$-functions, but still we can control the terms $\left|\nabla_{x} \mathbf{U}\right|,\left|\operatorname{div}_{x} \mathbf{U}\right|,\left|\mathbf{U} \cdot \nabla_{x} \mathbf{U}\right|$ and $\left|\nabla_{x} P(r)\right|$ by some constants.

Remark 3.4. This theorem applies to the already know results concerning strong solutions; in particular 
(i) if $\Omega$ is bounded, for local in time solutions see [16], and [15] for the global one;

(ii) if $\Omega=\mathbb{R}^{3}$, for local in time solution see for instance $[12,13]$, and [17] for the global one.

\section{Vanishing viscosity limit}

Unifying the two main results achieved in the previous sections, cf. Theorems 2.3 and 3.3, we conclude proving our last theorem: the solutions of the Navier-Stokes system converge in the zero viscosity limit to the strong solution of the Euler system with damping on the life span of the latter.

Theorem 4.1. Let $\Omega \subset \mathbb{R}^{N}, N=2,3$ be a domain with compact Lipschitz boundary and $\bar{\varrho}>0$ be a given far field density if $\Omega$ is unbounded. Suppose that $\gamma>\frac{N}{2}$ and let $\varrho_{R}, \boldsymbol{u}_{R}$ be a family of weak solutions to the Navier-Stokes system $(7)-(12)$ in

$$
(0, T) \times \Omega_{R}, \Omega_{R}=\Omega \cap B_{R},
$$

with initial data $\left\{\varrho_{R, 0}-\bar{\varrho}, \boldsymbol{m}_{R, 0}=\varrho_{R, 0} \boldsymbol{u}_{R, 0}\right\}_{R>0}$ such that

$$
\begin{aligned}
& \varrho_{R, 0}-\bar{\varrho} \rightarrow \varrho_{0}-\bar{\varrho} \quad \text { in } L^{2}+L^{\gamma}(\Omega) ; \\
& \boldsymbol{m}_{R, 0} \rightarrow \boldsymbol{m}_{0} \quad \text { in } L^{2}+L^{\frac{2 \gamma}{\gamma+1}}\left(\Omega ; \mathbb{R}^{N}\right) .
\end{aligned}
$$

Suppose that $\varrho_{0}>0,\left(\varrho_{0}-\bar{\varrho}, \frac{m_{0}}{\varrho_{0}}\right) \in H^{M}(\Omega), M>\frac{N}{2}+1$, and that $[r, \boldsymbol{U}] \in$ $H^{M}(\Omega)$ is the strong solution to the Euler system with damping with the same initial data.

Then

$$
\begin{aligned}
& \varrho_{R}-\bar{\varrho} \rightarrow r-\bar{\varrho} \quad \text { in } C_{\text {weak }}\left([0, T] ; L^{2}+L^{\gamma}(\Omega)\right) \text { and in } L^{1}((0, T) \times K) ; \\
& \boldsymbol{m}_{R}=\varrho_{R} \boldsymbol{u}_{R} \rightarrow r \boldsymbol{U} \quad \text { in } C_{\text {weak }}\left([0, T] ; L^{2}+L^{\frac{2 \gamma}{\gamma+1}}\left(\Omega ; \mathbb{R}^{N}\right)\right) \text { and in } L^{1}\left((0, T) \times K ; \mathbb{R}^{N}\right)
\end{aligned}
$$

for any compact $K \subset \Omega$.

Proof. Convergences (44), (45) follow easily from (23), repeating the same passages that we did in Sect. 2.3. In the proof of Theorem 2.3, we also showed that

$$
\begin{aligned}
& \varrho_{R}-\bar{\varrho} \rightarrow\left\langle\nu_{(\cdot, \cdot)} ; \varrho-\bar{\varrho}\right\rangle \quad \text { in } C_{\text {weak }}\left([0, T] ; L^{2}+L^{\gamma}(\Omega)\right) ; \\
& \mathbf{m}_{R} \rightarrow\left\langle\nu_{(\cdot, \cdot)} ; \mathbf{m}\right\rangle \quad \text { in } C_{\text {weak }}\left([0, T] ; L^{2}+L^{\frac{2 \gamma}{\gamma+1}}\left(\Omega ; \mathbb{R}^{N}\right)\right),
\end{aligned}
$$

where

$$
\begin{aligned}
& \nu_{t, x}:(t, x) \in(0, T) \times \Omega \rightarrow \mathcal{P}\left([0, \infty) \times \mathbb{R}^{N}\right), \\
& \nu \in L_{\text {weak }}^{\infty}\left((0, T) \times \Omega ; \mathcal{P}\left([0, \infty) \times \mathbb{R}^{N}\right)\right),
\end{aligned}
$$

is the Young measure associated to the sequence $\left\{\left(\varrho_{R}-\bar{\varrho}, \mathbf{m}_{R}\right)\right\}_{R>0}$ and also the dissipative measure-valued solution to the Euler system with damping. Then, since

$$
\nu_{0, x}=\delta_{\varrho_{0}(x), \mathbf{m}_{0}(x)} \quad \text { for a.e. } x \in \Omega
$$


we can apply Theorem 3.3 to get that

$$
\nu_{t, x}=\delta_{r(t, x), r \mathbf{U}(t, x)} \text { for a.e. }(t, x) \in(0, T) \times \Omega,
$$

and hence we obtain the claim.

\section{Acknowledgements}

This work was supported by the Einstein Foundation, Berlin. The author wishes to thank prof. Eduard Feireisl for the helpful advice and discussions.

Funding Open Access funding enabled and organized by Projekt DEAL.

Open Access. This article is licensed under a Creative Commons Attribution 4.0 International License, which permits use, sharing, adaptation, distribution and reproduction in any medium or format, as long as you give appropriate credit to the original author(s) and the source, provide a link to the Creative Commons licence, and indicate if changes were made. The images or other third party material in this article are included in the article's Creative Commons licence, unless indicated otherwise in a credit line to the material. If material is not included in the article's Creative Commons licence and your intended use is not permitted by statutory regulation or exceeds the permitted use, you will need to obtain permission directly from the copyright holder. To view a copy of this licence, visit http://creativecommons. org/licenses/by/4.0/.

Publisher's Note Springer Nature remains neutral with regard to jurisdictional claims in published maps and institutional affiliations.

\section{A. Young measures}

In this section we recall some useful definitions and results concerning the theory of Young measures.

Definition A.1. Let $Q \subseteq \mathbb{R}^{d}$ be an open set. The mapping $\nu: Q \rightarrow \mathcal{M}\left(\mathbb{R}^{m}\right)$ is said to be weak-* measurable, if for all $F \in L^{1}\left(Q ; C_{0}\left(\mathbb{R}^{m}\right)\right)$ the function

$$
Q \ni x \mapsto\left\langle\nu_{x}, F(x, \cdot)\right\rangle=\int_{\mathbb{R}^{m}} F(x, \lambda) d \nu_{x}(\lambda),
$$

is measurable.

Here and in the sequel we use the standard notation $\nu_{x}=\nu(x)$, as if measures $\nu_{x}$ were parametrized by $x$. For a weak-* measurable map $\nu$ we have

$$
\left\|\nu_{x}\right\|_{\mathcal{M}\left(\mathbb{R}^{m}\right)}=\sup _{\substack{f \in C_{0}\left(\mathbb{R}^{m}\right) \\\|f\|_{\infty} \leq 1}}\left|\left\langle\nu_{x}, f\right\rangle\right|,
$$

thus the function $x \mapsto\left\|\nu_{x}\right\|_{\mathcal{M}\left(\mathbb{R}^{m}\right)}$ is also measurable and we can define

$$
\|\nu\|_{L_{w}^{\infty}\left(\Omega ; \mathcal{M}\left(\mathbb{R}^{m}\right)\right)}=\underset{x \in Q}{\operatorname{ess} \sup }\left\|\nu_{x}\right\|_{\mathcal{M}\left(\mathbb{R}^{m}\right)} .
$$


Finally, we introduce the space

$$
\begin{gathered}
L_{\text {weak }}^{\infty}\left(Q ; \mathcal{M}\left(\mathbb{R}^{m}\right)\right)=\left\{\nu: Q \rightarrow \mathcal{M}\left(\mathbb{R}^{m}\right) ; \nu \text { weak-* measurable },\right. \\
\left.\|\nu\|_{L_{w}^{\infty}\left(\Omega ; \mathcal{M}\left(\mathbb{R}^{m}\right)\right)}<\infty\right\} .
\end{gathered}
$$

The following theorem holds.

Theorem A.2. Let $Q \subseteq \mathbb{R}^{n}$ be open. Let $\Phi \in\left(L^{1}\left(Q ; C_{0}\left(\mathbb{R}^{m}\right)\right)\right)^{*}$ be a linear bounded functional. Then there exists a unique $\nu \in L_{\text {weak }}^{\infty}\left(Q ; \mathcal{M}\left(\mathbb{R}^{m}\right)\right)$ such that, for all $F \in L^{1}\left(Q ; C_{0}\left(\mathbb{R}^{m}\right)\right)$,

$$
\Phi(F)=\int_{Q}\left\langle\nu_{x}, F(x)\right\rangle d x
$$

and

$$
\|\Phi\|_{\left(L^{1}\left(Q ; C_{0}\left(\mathbb{R}^{m}\right)\right)\right)^{*}}=\|\nu\|_{L_{w}^{\infty}\left(Q ; \mathcal{M}\left(\mathbb{R}^{m}\right)\right)} .
$$

Proof. See [14], Chapter 3, Theorem 2.11.

Let now $Q$ be a measurable set. Given a measurable sequence $\left\{\boldsymbol{z}^{R}\right\}_{R>0}$, we can construct the Young measure associated to it. First, for every $R$ we define the mapping

$$
\nu^{R}: Q \rightarrow \mathcal{M}\left(\mathbb{R}^{m}\right)
$$

defined for a.e. $y \in Q$ by

$$
\nu_{y}^{R}=\delta_{z^{R}(y)},
$$

where $\delta_{a}$ is the Dirac measure supported at $a \in \mathbb{R}^{m}$. Hence, for every $\psi \in$ $L^{1}\left(Q ; C_{0}\left(\mathbb{R}^{m}\right)\right)$ the function

$$
y \mapsto\left\langle\nu_{y}^{R}, \psi(y)\right\rangle
$$

is measurable since it is integrable; indeed

$$
\left\langle\nu_{y}^{R}, \psi(y)\right\rangle=\int_{\mathbb{R}^{m}} \psi(y, \cdot) d \nu_{y}^{R}=\int_{\mathbb{R}^{m}} \psi(y, \cdot) d \delta_{z^{R}(y)}=\psi\left(y, z^{R}(y)\right),
$$

and then

$$
\int_{Q}\left|\left\langle\nu_{y}^{R}, \psi(y)\right\rangle\right| d y \leq \int_{Q} \sup _{\lambda \in \mathbb{R}^{m}}|\psi(y, \lambda)| d y=\|\psi\|_{L^{1}\left(Q ; C_{0}\left(\mathbb{R}^{m}\right)\right)} .
$$

The mapping $\nu^{R}$ is then weakly-* measurable with

$$
\left\|\nu^{R}\right\|_{L_{w}^{\infty}\left(Q ; \mathcal{M}\left(\mathbb{R}^{m}\right)\right)}=\underset{y \in Q}{\operatorname{ess} \sup _{0}}\left\|\nu_{y}^{R}\right\|_{\mathcal{M}\left(\mathbb{R}^{m}\right)}=\left\|\delta_{z^{R}(y)}\right\|_{\mathcal{M}\left(\mathbb{R}^{m}\right)}=1 .
$$

Therefore, $\left\{\nu^{R}\right\}_{R>0}$ is uniformly bounded in $L_{\text {weak }}^{\infty}\left(Q ; \mathcal{M}\left(\mathbb{R}^{m}\right)\right)$, which by Theorem A.2 is the dual space of the separable space $L^{1}\left(Q ; C_{0}\left(\mathbb{R}^{m}\right)\right)$; we can apply the Banach-Alaoglu theorem to find a subsequence, not relabeled, and $\nu \in L_{\text {weak }}^{\infty}\left(Q ; \mathcal{M}\left(\mathbb{R}^{m}\right)\right)$ such that

$$
\nu^{R} \stackrel{*}{\rightarrow} \nu \quad \text { in } L_{\text {weak }}^{\infty}\left(Q ; \mathcal{M}\left(\mathbb{R}^{m}\right)\right) .
$$

This means that for all $\psi \in L^{1}\left(Q ; C_{0}\left(\mathbb{R}^{4}\right)\right)$

$$
\int_{Q} \psi\left(y, \boldsymbol{z}^{R}(y)\right) d y=\int_{Q}\left\langle\nu_{y}^{R}, \psi(y)\right\rangle d y \rightarrow \int_{Q}\left\langle\nu_{y}, \psi(y)\right\rangle d y \quad \text { as } R \rightarrow \infty .
$$


If we now choose $\psi(y, \lambda)=g(y) \varphi(\lambda)$ with $g \in L^{1}(Q), \varphi \in C_{0}\left(\mathbb{R}^{m}\right)$, the last limit tells us that

$$
\int_{Q} g(t, x) \varphi\left(z^{R}(y)\right) d y=\int_{Q} g(y)\left\langle\nu_{y}^{R}, \varphi\right\rangle d y \rightarrow \int_{Q} g(y)\left\langle\nu_{y}, \varphi\right\rangle d y \quad \text { as } R \rightarrow \infty .
$$

Then, for every $\varphi \in C_{0}\left(\mathbb{R}^{m}\right)$, knowing that

$$
\varphi\left(\boldsymbol{z}^{R}\right) \stackrel{*}{\rightarrow} \bar{\varphi} \text { in } L^{\infty}(Q),
$$

we can deduce that

$$
\bar{\varphi}(y)=\left\langle\nu_{y}, \varphi\right\rangle \quad \text { for a.e. } y \in Q .
$$

From the weak-* lower semi-continuity of the norm we also have that

$$
\left\|\nu_{y}\right\|_{\mathcal{M}\left(\mathbb{R}^{m}\right)} \leq \liminf _{R \rightarrow \infty}\left\|\nu_{y}^{R}\right\|_{\mathcal{M}\left(\mathbb{R}^{m}\right)}=1 \quad \text { for a.e. } y \in Q .
$$

What we proved is the first statement of the following theorem.

Theorem A.3. Let $Q \subset \mathbb{R}^{d}$ be a measurable set and let $\boldsymbol{z}^{R}: Q \rightarrow \mathbb{R}^{m}, R>0$, be a sequence of measurable functions. Then there exists a subsequence, still denoted by $z^{R}$, and a measure-valued function $\nu$ with the following properties:

1. $\nu \in L_{\text {weak }}^{\infty}\left(Q ; \mathcal{M}\left(\mathbb{R}^{m}\right)\right),\left\|\nu_{y}\right\|_{\mathcal{M}\left(\mathbb{R}^{m}\right)} \leq 1$, for a.e. $y \in Q$ and we have for every $\varphi \in C_{0}\left(\mathbb{R}^{m}\right)$, as $R \rightarrow \infty$,

$$
\varphi\left(z^{R}\right) \stackrel{*}{\rightarrow} \bar{\varphi} \text { in } L^{\infty}(Q), \quad \bar{\varphi}(y)=\left\langle\nu_{y}, \varphi\right\rangle, \text { for a.e. } y \in Q
$$

2. moreover, if

$$
\lim _{k \rightarrow \infty} \sup _{R>0} \operatorname{meas}\left\{y \in Q \cap B_{r} ;\left|z^{R}(y)\right| \geq k\right\}=0
$$

for every $r>0$, where $B_{r} \equiv\{y \in Q ;|y| \leq r\}$, then

$$
\left\|\nu_{y}\right\|_{\mathcal{M}\left(\mathbb{R}^{m}\right)}=1 \text { for a.e. } y \in Q \text {; }
$$

3. Let $\Psi:[0, \infty) \rightarrow \mathbb{R}$ be a Young function satisfying the $\Delta_{2}$-condition. If condition (47) holds and if we have for some continuous function $\tau$ : $\mathbb{R}^{m} \rightarrow \mathbb{R}$

$$
\sup _{R>0} \int_{Q} \Psi\left(\left|\tau\left(z^{R}\right)\right|\right) d y<\infty
$$

then

$$
\tau\left(z^{R}\right) \stackrel{*}{\rightarrow} \bar{\tau} \text { in the Orlicz space } L_{\Psi}(Q), \quad \bar{\tau}(y)=\left\langle\nu_{y}, \tau\right\rangle \text { for a.e. } y \in Q .
$$

Proof. See [14], Chapter 4, Theorem 2.1.

Remark A.4. If $\boldsymbol{z}^{R}$ are uniformly bounded in $L^{p}\left(Q ; \mathbb{R}^{m}\right)$ for some $p \in[1, \infty)$, the condition (47) is satisfied. Indeed, denoting $A_{k}^{R} \equiv\left\{y \in Q \cap B_{r} ;\left|z^{R}(y)\right| \geq\right.$ $k$, we have

$$
\left|A_{k}^{R}\right| k^{p} \leq \int_{A_{k}^{R}}\left|\boldsymbol{z}^{R}(y)\right|^{p} d y \leq \int_{Q}\left|\boldsymbol{z}^{R}(y)\right|^{p} d y \leq c .
$$

Since $c$ is independent of both $R$ and $k$, we obtain

$$
\sup _{R>0}\left|A_{k}^{R}\right| \leq \frac{c}{k^{p}}
$$

which implies (47). 
The following two lemma justify the introduction of the concentration measures and dissipation defect in Definition 2.2

Lemma A.5. Let $\left\{\boldsymbol{z}^{R}\right\}_{R>0}, \boldsymbol{z}^{R}: Q \rightarrow \mathbb{R}^{m}$ be a sequence of measurable functions generating a Young measure $\nu \in L_{\text {weak }}^{\infty}\left(Q ; \mathcal{M}\left(\mathbb{R}^{m}\right)\right)$, where $Q$ is a measurable set in $\mathbb{R}^{d}$. For every continuous function $H: \mathbb{R}^{m} \rightarrow \mathbb{R}$ such that

$$
\left\|H\left(\boldsymbol{z}^{R}\right)\right\|_{L^{1}(Q)} \leq c, \quad \text { uniformly in } R,
$$

$\left\langle\nu_{y} ; H\right\rangle$ is finite for a.e. $y \in Q$.

Proof. Without loss of generality, we can consider $|H|$ or, equivalently, assume that $H \geq 0$. We take a family of cut-off functions

$$
T_{k}(z)=\min \{z, k\} ;
$$

Then $T_{k}(H) \in C_{0}\left(\mathbb{R}^{m}\right)$ and thus

$$
T_{k}\left(H\left(z^{R}\right)\right) \stackrel{*}{\rightarrow} \overline{T_{k}(H)} \quad \text { in } L^{\infty}(Q)
$$

with

$$
\overline{T_{k}(H)}(y)=\left\langle\nu_{t, x}, T_{k}(H)\right\rangle \quad \text { for a.e. } y \in Q .
$$

On the other hand we have that

$$
T_{k}(H)(\lambda) \nearrow H(\lambda), \text { for any } \lambda \in \mathbb{R}^{m} \text { as } k \rightarrow \infty ;
$$

by monotone convergence theorem, we have that

$$
\left\langle\nu_{y}, T_{k}(H)\right\rangle=\int_{\mathbb{R}^{m}} T_{k}(H)(\lambda) d \nu_{y}(\lambda) \rightarrow \int_{\mathbb{R}^{m}} H(\lambda) d \nu_{y}(\lambda) \quad \text { for a.e. } y \in Q \text { as } k \rightarrow \infty \text {. }
$$

Hence $H$ is $\nu_{y}$-integrable but the integral can also be infinite. However, by the weak-* lower semi-continuity of the norm

$$
\|\left\langle\nu_{(\cdot)}, T_{k}(H)\left\|_{L^{1}(Q)} \leq \liminf _{R \rightarrow \infty}\right\| T_{k}\left(H\left(\boldsymbol{z}^{R}\right)\right)\left\|_{L^{1}(Q)} \leq \liminf _{R \rightarrow \infty}\right\| H\left(\boldsymbol{z}^{R}\right) \|_{L^{1}(Q)} \leq c,\right.
$$

uniformly in $k$. Then, since

(i) $\lim _{k \rightarrow \infty}\left\langle\nu_{y} ; T_{k}(H)\right\rangle=\left\langle\nu_{y} ; H\right\rangle$ for a.e. $y \in Q$;

(ii) $\sup _{k \in \mathbb{N}}\left\|\left\langle\nu_{(\cdot)} ; T_{k}(H)\right\rangle\right\|_{L^{1}(Q)} \leq c$,

applying Fatou's lemma we get that $\left\|\left\langle\nu_{(\cdot)} ; H\right\rangle\right\|_{L^{1}(Q)} \leq c$. Then $\left\langle\nu_{y} ; H\right\rangle$ is finite for a.e. $y \in Q$.

Lemma A.6. Let $\left\{\boldsymbol{z}^{R}\right\}_{R>0}, \boldsymbol{z}^{R}: Q \subset \mathbb{R}^{d} \rightarrow \mathbb{R}^{m}$ be a sequence of measurable functions generating a Young measure $\nu \in L_{\text {weak }}^{\infty}\left(Q ; \mathcal{M}\left(\mathbb{R}^{m}\right)\right)$, where $Q$ is a measurable set in $\mathbb{R}^{d}$. Let

$$
G: \mathbb{R}^{m} \rightarrow[0, \infty)
$$

be a continuous function such that

$$
\left\|G\left(\boldsymbol{z}^{R}\right)\right\|_{L^{1}(Q)}<\infty, \quad \text { uniformly in } R,
$$

and let $F$ be continuous such that

$$
F: \mathbb{R}^{m} \rightarrow \mathbb{R}, \quad|F(\boldsymbol{z})| \leq G(\boldsymbol{z}) \text { for all } \boldsymbol{z} \in \mathbb{R}^{m} .
$$

Denote

$$
\mu_{F_{\infty}}=\mu_{\tilde{F}}-\left\langle\nu_{y}, F(\boldsymbol{v})\right\rangle d y, \quad \mu_{G_{\infty}}=\mu_{\tilde{G}}-\left\langle\nu_{y}, G(\boldsymbol{v})\right\rangle d y,
$$


where $\mu_{\tilde{F}}, \mu_{\tilde{G}} \in \mathcal{M}(Q)$ are the weak-* limits of $\left\{F\left(\boldsymbol{z}^{R}\right)\right\}_{R>0},\left\{G\left(\boldsymbol{z}^{R}\right)\right\}_{R>0}$ in $\mathcal{M}(Q)$. Then

$$
\left|F_{\infty}\right| \leq G_{\infty}
$$

Proof. We have seen that the Young measure $\left\{\nu_{y}\right\}_{y \in Q}$ is such that for all $\psi \in L^{1}\left(Q ; C_{0}\left(\mathbb{R}^{m}\right)\right)$

$$
\int_{Q} \psi\left(y, z^{R}(y)\right) d y \rightarrow \int_{Q}\left\langle\nu_{y}, \psi(y)\right\rangle d y=\int_{Q} \int_{\mathbb{R}^{m}} \psi(y, \lambda) d \nu_{y}(\lambda) d y,
$$

as $R \rightarrow \infty$. Now, from the fact that

$$
\begin{aligned}
& \mu_{F\left(\boldsymbol{z}^{R}\right)} \stackrel{*}{\rightarrow} \mu_{\tilde{F}} \quad \text { in } \mathcal{M}(Q), \\
& \mu_{G\left(\boldsymbol{z}^{R}\right)} \stackrel{*}{\rightarrow} \mu_{\tilde{G}} \quad \text { in } \mathcal{M}(Q),
\end{aligned}
$$

we have that for all $\varphi \in C_{0}(Q)$

$$
\begin{aligned}
\left\langle\mu_{\tilde{F}}, \varphi\right\rangle & =\lim _{R \rightarrow \infty} \int_{Q} F\left(\boldsymbol{z}^{R}\right) \varphi d y \\
& =\lim _{R \rightarrow \infty} \int_{\left\{\left|\boldsymbol{z}^{R}\right| \leq M\right\}} F\left(\boldsymbol{z}^{R}\right) \varphi d y+\lim _{R \rightarrow \infty} \int_{\left\{\left|\boldsymbol{z}^{R}\right|>M\right\}} F\left(\boldsymbol{z}^{R}\right) \varphi d y, \\
\left\langle\mu_{\tilde{G}}, \varphi\right\rangle & =\lim _{R \rightarrow \infty} \int_{Q} G\left(\boldsymbol{z}^{R}\right) \varphi d y \\
& =\lim _{R \rightarrow \infty} \int_{\left\{\left|\boldsymbol{z}^{R}\right| \leq M\right\}} G\left(\boldsymbol{z}^{R}\right) \varphi d y+\lim _{R \rightarrow \infty} \int_{\left\{\left|\boldsymbol{z}^{R}\right|>M\right\}} G\left(\boldsymbol{z}^{R}\right) \varphi d y .
\end{aligned}
$$

Now, we can write

$$
\int_{\left\{\left|\boldsymbol{z}^{R}\right| \leq M\right\}} F\left(\boldsymbol{z}^{R}(y)\right) \varphi(y) d y=\int_{Q} \psi\left(y, \boldsymbol{z}^{R}(y)\right) d y,
$$

with

$$
\psi(y, \lambda)=F(\lambda) \varphi(y) \chi_{\{|\lambda| \leq M\}} ;
$$

then, we have that $\psi \in L^{1}\left(Q ; C_{0}\left(\mathbb{R}^{m}\right)\right)$; indeed, calling $K=\operatorname{supp}(\varphi)$ we have $\int_{Q}\|\psi(y, \cdot)\|_{C_{0}\left(\mathbb{R}^{m}\right)} d y=\int_{K}|\varphi(y)| \sup _{|\lambda| \leq M}|F(\lambda)| \leq|K| \sup _{y \in K}|\varphi(y)| \sup _{|\lambda| \leq M}|F(\lambda)| \leq c$, since both $\varphi$ and $F$ are continuous functions and so they admit maximum on compact sets. Then, for what we have told previously, we have

$$
\begin{aligned}
\lim _{R \rightarrow \infty} \int_{\left\{\left|\boldsymbol{z}^{R}\right| \leq M\right\}} F\left(\boldsymbol{z}^{R}\right) \varphi d y & =\lim _{R \rightarrow \infty} \int_{Q} \psi\left(y, \boldsymbol{z}^{R}(y)\right) d y \\
& =\int_{Q}\left\langle\nu_{y}, \psi(y)\right\rangle d y=\int_{Q} \int_{\mathbb{R}^{m}} \psi(y, \lambda) d \nu_{y}(\lambda) d y \\
& =\int_{Q} \int_{\{|\lambda| \leq M\}} F(\lambda) \varphi(y) d \nu_{y}(\lambda) d y .
\end{aligned}
$$

Applying now Lebesgue theorem we have

$$
\lim _{M \rightarrow \infty}\left(\lim _{R \rightarrow \infty} \int_{\left\{\left|\boldsymbol{z}^{R}\right| \leq M\right\}} F\left(\boldsymbol{z}^{R}\right) \varphi d y\right)=\int_{Q}\left(\int_{\mathbb{R}^{m}} F(\lambda) d \nu_{y}(\lambda)\right) \varphi d y
$$




$$
=\int_{Q}\left\langle\nu_{y} ; F\right\rangle \varphi d y
$$

Similarly

$$
\lim _{M \rightarrow \infty}\left(\lim _{R \rightarrow \infty} \int_{\left\{\left|z^{R}\right| \leq M\right\}} G\left(z^{R}\right) \varphi d y\right)=\int_{Q}\left\langle\nu_{y} ; G\right\rangle \varphi d y .
$$

Then, we deduce

$$
\begin{aligned}
& \left\langle\mu_{F_{\infty}}, \varphi\right\rangle=\lim _{M \rightarrow \infty}\left(\lim _{R \rightarrow \infty} \int_{\left\{\left|\boldsymbol{z}^{R}\right|>M\right\}} F\left(\boldsymbol{z}^{R}\right) \varphi d y\right), \\
& \left\langle\mu_{G_{\infty}}, \varphi\right\rangle=\lim _{M \rightarrow \infty}\left(\lim _{R \rightarrow \infty} \int_{\left\{\left|\boldsymbol{z}^{R}\right|>M\right\}} G\left(\boldsymbol{z}^{R}\right) \varphi d y\right) .
\end{aligned}
$$

Then, from condition $|F| \leq G$ we obtain what we wanted to prove.

\section{A.1. Young measure for the compressible Euler system with damping}

Our next goal is to adapt the abstract machinery presented in previous subsection in order to justify the definition of a dissipative measure-valued solution for the compressible Euler system. To this end, it is enough to take

- $Q=(0, T) \times \Omega \subset \mathbb{R}^{N+1}$;

- $m=N+1$;

- $\boldsymbol{z}^{R}=\left(\varrho_{R}-\bar{\varrho}, \mathbf{m}_{R}\right)$,

where $\left(\varrho_{R}-\bar{\varrho}, \mathbf{m}_{R}=\varrho_{R} \mathbf{u}_{R}\right)$ are the weak solutions of the Navier-Stokes system (14), (15).

First of all, notice that condition (47) is satisfied for $\boldsymbol{z}^{R}=\left(\varrho_{R}-\bar{\varrho}, \mathbf{m}_{R}\right)$; indeed, introducing the sets $A_{k}^{R} \equiv\left\{y \in Q \cap B_{r} ;\left|z^{R}(y)\right| \geq k\right\}$ we have, for $y \in A_{k}^{R}$

$$
\begin{aligned}
k \leq\left|\left(\varrho_{R}-\bar{\varrho}, \mathbf{m}_{R}\right)(y)\right| \leq & \left|\varrho_{R}(y)\right|+\left|\mathbf{m}_{R}(y)\right| \\
\leq & \left|\left[\varrho_{R}-\bar{\varrho}\right]_{e s s}(y)\right|+\left|\left[\varrho_{R}-\bar{\varrho}\right]_{\text {res }}(y)\right| \\
& +\left|\left[\mathbf{m}_{R}\right]_{\text {ess }}(y)\right|+\left|\left[\mathbf{m}_{R}\right]_{\text {res }}(y)\right|,
\end{aligned}
$$

and hence at least one of the terms on the last line must be $\geq \frac{k}{4}$ so that

$$
\begin{aligned}
A_{k}^{R} \subseteq & \underbrace{\left\{y \in Q \cap B_{r} ;\left|\left[\varrho_{R}-\bar{\varrho}\right]_{e s s}(y)\right| \geq \frac{k}{4}\right\}}_{\equiv A_{k, 1}^{R}} \cup \underbrace{\left\{y \in Q \cap B_{r} ;\left|\left[\varrho_{R}-\bar{\varrho}\right]_{r e s}(y)\right| \geq \frac{k}{4}\right\}}_{\equiv A_{k, 2}^{R}} . \\
& \cup \underbrace{\left\{y \in Q \cap B_{r} ;\left|\left[\mathbf{m}_{R}\right]_{\text {ess }}(y)\right| \geq \frac{k}{4}\right\}}_{\equiv A_{k, 3}^{R}} \cup \underbrace{\left\{y \in Q \cap B_{r} ;\left|\left[\mathbf{m}_{R}\right]_{\text {res }}(y)\right| \geq \frac{k}{4}\right\}}_{\equiv A_{k, 4}^{R}} .
\end{aligned}
$$

For $k$ large enough $(k \geq 4)$, we have

$$
\begin{aligned}
\left|A_{k}^{R}\right| k & \leq 4 \sum_{i=1}^{4}\left|A_{k, i}^{R}\right| \frac{k}{4} \\
& \lesssim\left|A_{k, 1}^{R}\right|\left(\frac{k}{4}\right)^{2}+\left|A_{k, 2}^{R}\right|\left(\frac{k}{4}\right)^{\gamma}+\left|A_{k, 3}^{R}\right|\left(\frac{k}{4}\right)^{2}+\left|A_{k, 4}^{R}\right|\left(\frac{k}{4}\right)^{\frac{2 \gamma}{\gamma+1}}
\end{aligned}
$$




$$
\begin{aligned}
\leq & \int_{A_{k, 1}^{R}}\left|\left[\varrho_{R}-\bar{\varrho}\right]_{e s s}(y)\right|^{2} d y+\int_{A_{k, 2}^{R}}\left|\left[\varrho_{R}-\bar{\varrho}\right]_{r e s}(y)\right|^{\gamma} d y \\
& +\int_{A_{k, 3}^{R}}\left|\left[\mathbf{m}_{R}\right]_{e s s}(y)\right|^{2} d y+\int_{A_{k, 4}^{R}}\left|\left[\mathbf{m}_{R}\right]_{r e s}(y)\right|^{\frac{2 \gamma}{\gamma+1}} d y \\
\leq & \left\|\left[\varrho_{R}-\bar{\varrho}\right]_{e s s}\right\|_{L^{2}(Q)}+\left\|\left[\varrho_{R}-\bar{\varrho}\right]_{r e s}\right\|_{L^{\gamma}(Q)}+\left\|\left[\mathbf{m}_{R}\right]_{e s s}\right\|_{L^{2}(Q)} \\
& +\left\|\left[\mathbf{m}_{R}\right]_{r e s}\right\|_{L^{\frac{2 \gamma}{\gamma+1}}(Q)} \\
\leq & c\left(E_{0}\right),
\end{aligned}
$$

where in particular the constant $c\left(E_{0}\right)$ is independent of $k$ and $R$ so that

$$
\sup _{R>0}\left|A_{k}^{R}\right| \leq \frac{c}{k}
$$

which implies (47).

The Young measure in our case is then a parametrized family of probability measures supported on the set $[0, \infty) \times \mathbb{R}^{N}$, since the densities are supposed to be non-negative:

$$
\begin{aligned}
& \nu_{t, x}:(t, x) \in(0, T) \times \Omega \rightarrow \mathcal{P}\left([0, \infty) \times \mathbb{R}^{N}\right), \\
& \nu \in L_{\text {weak }}^{\infty}\left((0, T) \times \Omega ; \mathcal{P}\left([0, \infty) \times \mathbb{R}^{N}\right)\right) .
\end{aligned}
$$

It is also easy to check that $\Psi(t)=t^{p}$ with $p>1$ are Young functions that satisfy the $\Delta_{2}$-condition with the constant $2^{p}$, and in that case $L_{\Psi}(Q)=$ $L^{p}(Q)$. Thus,

1. first, we can take $\Psi(t)=t^{2}$ and $\tau_{1}(\boldsymbol{z})=z_{1} \chi\left(z_{1}+\bar{\varrho}\right)$, where $\boldsymbol{z}=$ $\left(z_{1}, z_{2}, z_{3}, z_{4}\right)$ in our case, to notice that condition (48) is equivalent in requiring that $\left[\varrho_{R}-\bar{\varrho}\right]_{\text {ess }}$ are uniformly bounded in $L^{2}((0, T) \times \Omega)$ which is true from (28). Then we obtain

$$
\left\langle\nu_{t, x} ; \tau_{1}\right\rangle=f_{\varrho-\bar{\varrho}}(t, x) \text { for a.e. }(t, x) \in(0, T) \times \Omega ;
$$

also, taking $\Psi(t)=t^{\gamma}$ and $\tau_{2}(\boldsymbol{z})=z_{1}\left(1-\chi\left(z_{1}+\bar{\varrho}\right)\right)$, condition (48) is equivalent in requiring that $\left[\varrho_{R}-\bar{\varrho}\right]_{\text {res }}$ are uniformly bounded in $L^{\gamma}((0, T) \times \Omega)$ which is true from $(29)$. Then we obtain

$$
\left\langle\nu_{t, x} ; \tau_{2}\right\rangle=g_{\varrho-\bar{\varrho}}(t, x) \text { for a.e. }(t, x) \in(0, T) \times \Omega .
$$

Unifying the two results we get

$$
\left\langle\nu_{t, x} ; \tau_{1}+\tau_{2}\right\rangle=(\varrho-\bar{\varrho})(t, x) \text { for a.e. }(t, x) \in(0, T) \times \Omega,
$$

where $\varrho-\bar{\varrho}$ is the weak limit of the densities found in (32). We will write $\left\langle\nu_{t, x} ; \varrho-\bar{\varrho}\right\rangle=(\varrho-\bar{\varrho})(t, x)$ for almost every $(t, x) \in(0, T) \times \Omega$ just to make the notation readable;

2. secondly, we can take $\Psi(t)=t^{2}$ and $\tau_{1}(\boldsymbol{z})=z_{i} \chi\left(z_{1}+\bar{\varrho}\right)$ with $i=2,3,4$ to see that condition (48) is equivalent in requiring that each component of $\left[\mathbf{m}_{R}\right]_{\text {ess }}$ is uniformly bounded in $L^{2}((0, T) \times \Omega)$ which is true from $(30)$. Also, choosing $\Psi(t)=t^{\frac{2 \gamma}{\gamma+1}}$ and $\tau_{2}(\boldsymbol{z})=z_{i}\left(1-\chi\left(z_{1}+\bar{\varrho}\right)\right)$ with $i=2,3,4$, condition (48) is equivalent in requiring that each component of $\left[\mathbf{m}_{R}\right]_{\text {res }}$ 
is uniformly bounded in $L^{\frac{2 \gamma}{\gamma+1}}((0, T) \times \Omega)$ which is true from $(31)$. Then we obtain

$$
\left\langle\nu_{t, x} ; \tau_{1}+\tau_{2}\right\rangle=m_{i}(t, x) \text { for a.e. }(t, x) \in(0, T) \times \Omega
$$

which we will write $\left\langle\nu_{t, x} ; \mathbf{m}\right\rangle=\mathbf{m}(t, x)$ for almost every $(t, x) \in(0, T) \times \Omega$, with $\mathbf{m}$ the weak limit of the momenta found in (33).

Moreover, due to Lemma A.5, it makes sense to introduce the following new measures:

$$
\begin{aligned}
\mu_{\mathbb{M}_{\infty}} & =\mu_{\{\mathbb{M}\}}-\left\langle\nu_{(\cdot, \cdot)} ; \frac{\mathbf{m} \otimes \mathbf{m}}{\varrho}\right\rangle d x d t \\
\mu_{p_{\infty}} & =\mu_{\{p\}-p(\bar{\varrho})}-\left\langle\nu_{(\cdot, \cdot)} ; p(\varrho)-p(\bar{\varrho})\right\rangle d x d t \\
\mu_{\sigma_{\infty}} & =\mu_{\operatorname{trace}\{\mathbb{M}\}}-\left\langle\nu_{(\cdot, \cdot)} ; \frac{|\mathbf{m}|^{2}}{\varrho}\right\rangle d x d t \\
\mu_{E_{\infty}} & =\mu_{\{E\}}-\left\langle\nu_{(\cdot, \cdot)} ; \frac{1}{2} \frac{|\mathbf{m}|^{2}}{\varrho}+P(\varrho)-P^{\prime}(\bar{\varrho})(\varrho-\bar{\varrho})-P(\bar{\varrho})\right\rangle d x d t .
\end{aligned}
$$

Now, revisiting the momentum equation (35) and using the fact that

$$
\operatorname{div}_{x} \varphi=\mathbb{I}: \nabla_{x} \varphi
$$

we get

$$
\begin{aligned}
\int_{\Omega} \mathbf{m} \cdot \boldsymbol{\varphi}(\tau, \cdot) d x-\int_{\Omega} \mathbf{m}_{0} \cdot \boldsymbol{\varphi}(0, \cdot) d x \\
=\int_{0}^{\tau} \int_{\Omega}\left[\mathbf{m} \cdot \partial_{t} \boldsymbol{\varphi}+\left(\left\langle\nu_{t, x} ; \frac{\mathbf{m} \otimes \mathbf{m}}{\varrho}\right\rangle+\mathbb{M}_{\infty}\right): \nabla_{x} \boldsymbol{\varphi}\right] d x d t \\
\quad+\int_{0}^{\tau} \int_{\Omega}\left[\left(\left\langle\nu_{t, x} ; p(\varrho)\right\rangle+p_{\infty}\right) \mathbb{I}: \nabla_{x} \boldsymbol{\varphi}-a \mathbf{m} \cdot \boldsymbol{\varphi}\right] d x d t
\end{aligned}
$$

for all $\tau \in[0, T)$ and for all $\varphi \in C_{c}^{1}\left([0, T] \times \bar{\Omega} ; \mathbb{R}^{N}\right),\left.\boldsymbol{\varphi} \cdot \mathbf{n}\right|_{\partial \Omega}=0$, which can be rewritten as

$$
\begin{aligned}
\int_{\Omega} \mathbf{m} \cdot \boldsymbol{\varphi}(\tau, \cdot) d x-\int_{\Omega} \mathbf{m}_{0} \cdot \boldsymbol{\varphi}(0, \cdot) d x= & \int_{0}^{\tau} \int_{\Omega}\left[\mathbf{m} \cdot \partial_{t} \boldsymbol{\varphi}+\left\langle\nu_{t, x} ; \frac{\mathbf{m} \otimes \mathbf{m}}{\varrho}\right\rangle: \nabla_{x} \boldsymbol{\varphi}\right] d x d t \\
& +\int_{0}^{\tau} \int_{\Omega}\left[\left\langle\nu_{t, x} ; p(\varrho)\right\rangle \operatorname{div} x \boldsymbol{\varphi}-a \mathbf{m} \cdot \boldsymbol{\varphi}\right] d x d t \\
& +\int_{0}^{\tau} \int_{\Omega} \nabla_{x} \boldsymbol{\varphi}: d \mu_{m},
\end{aligned}
$$

for all $\tau \in[0, T)$ and for all $\varphi \in C_{c}^{1}\left([0, T] \times \bar{\Omega} ; \mathbb{R}^{N}\right),\left.\boldsymbol{\varphi} \cdot \mathbf{n}\right|_{\partial \Omega}=0$, where $\mu_{m}=\mathbb{M}_{\infty}+p_{\infty} \mathbb{I} \in \mathcal{M}\left([0, T] \times \bar{\Omega} ; \mathbb{R}^{N} \times \mathbb{R}^{N}\right)$ is a tensor-valued measure. 
Similarly, from (36) we get

$$
\begin{aligned}
\int_{\Omega} & {\left[\left\langle\nu_{\tau, x} ; \frac{1}{2} \frac{|\mathbf{m}|^{2}}{\varrho}+P(\varrho)-P^{\prime}(\bar{\varrho})(\varrho-\bar{\varrho})-P(\bar{\varrho})\right\rangle+E_{\infty}(\tau)\right] d x } \\
& +a \int_{0}^{\tau} \int_{\Omega}\left[\left\langle\nu_{t, x} ; \frac{|\mathbf{m}|^{2}}{\varrho}\right\rangle+\sigma_{\infty}\right] d x d t \\
& \leq \int_{\Omega}\left[\left\langle\nu_{0, x} ; \frac{1}{2} \frac{|\mathbf{m}|^{2}}{\varrho}+P(\varrho)-P^{\prime}(\bar{\varrho})(\varrho-\bar{\varrho})(\varrho-\bar{\varrho})-P(\bar{\varrho})\right\rangle+E_{\infty}(0)\right] d x
\end{aligned}
$$

for a.e. $\tau \in(0, T)$, which can be rewritten as

$$
\begin{aligned}
& \int_{\Omega}\left\langle\nu_{\tau, x} ; \frac{1}{2} \frac{|\mathbf{m}|^{2}}{\varrho}+P(\varrho)-P^{\prime}(\bar{\varrho})(\varrho-\bar{\varrho})-P(\bar{\varrho})\right\rangle d x \\
& \quad+a \int_{0}^{\tau} \int_{\Omega}\left\langle\nu_{t, x} ; \frac{|\mathbf{m}|^{2}}{\varrho}\right\rangle d x d t+\mathcal{D}(\tau) \\
& \quad \leq \int_{\Omega}\left\langle\nu_{0, x} ; \frac{1}{2} \frac{|\mathbf{m}|^{2}}{\varrho}+P(\varrho)-P^{\prime}(\bar{\varrho})(\varrho-\bar{\varrho})-P(\bar{\varrho})\right\rangle d x
\end{aligned}
$$

for a.e. $\tau \in(0, T)$, with $\mathcal{D} \in L^{\infty}(0, T)$ such that

$$
\mathcal{D}(\tau)=\int_{\Omega} E_{\infty}(\tau) d x+a \int_{0}^{\tau} \int_{\Omega} \sigma_{\infty} d x d t
$$

We also have that

$$
\int_{0}^{\tau} \int_{\Omega} d\left|\mu_{m}\right| \lesssim \int_{0}^{\tau} \mathcal{D}(t) d t
$$

for a.e. $\tau \in(0, T)$. Indeed,

$$
\begin{aligned}
\int_{0}^{\tau} \int_{\Omega} d\left|\mu_{m}\right| & \leq \sum_{i, j=1}^{N} \int_{0}^{\tau} \int_{\Omega}\left|\left(\mathbb{M}_{\infty}\right)_{i, j}\right| d x d t+\sum_{i, j=1}^{N} \int_{0}^{\tau} \int_{\Omega}\left|p_{\infty}\right| \delta_{i, j} d x d t \\
& =\sum_{i, j=1}^{N} \int_{0}^{\tau} \int_{\Omega}\left|\left(\mathbb{M}_{\infty}\right)_{i, j}\right| d x d t+N \int_{0}^{\tau} \int_{\Omega}\left|p_{\infty}\right| d x d t .
\end{aligned}
$$

Now it is sufficient to apply Lemma A.6 with $F=p(\varrho)-p(\bar{\varrho}), G=P(\varrho)-$ $P^{\prime}(\bar{\varrho})(\varrho-\bar{\varrho})-P(\bar{\varrho})$ first and $F=\frac{m_{i} m_{j}}{\varrho}, G=\frac{|\mathbf{m}|^{2}}{\varrho}$ then, to get

$$
\int_{0}^{\tau} \int_{\Omega} d\left|\mu_{m}\right| \lesssim \int_{0}^{\tau} \int_{\Omega} E_{\infty} d x d t \leq \int_{0}^{\tau} \mathcal{D}(t) d t .
$$

\section{References}

[1] Alibert, J.J., Bouchitté, G.: Non-uniform integrability and generalized Young measures. J. Convex Anal. 4, 129-147 (1997)

[2] Březina, J., Mácha, V.: Inviscid limit for the compressible Euler system with non-local interactions. J. Differ. Equ. 267(7), 4410-4428 (2019) 
[3] Chen, G.-Q.G., Glimm, J.: Kolmogorov-type theory of compressible turbolence and inviscid limit of the Navier-Stokes equations in $\mathbb{R}^{3}$. Phys. D Nonlinear Phenom. 2019, 400 (2019)

[4] Di Perna, R.J., Majda, A.J.: Oscillations and concentrations in weak solutions of the incompressible fluid equations. Comm. Math. Phys. 108(4), 667-689 (1987)

[5] Feireisl, E., Gwiazda, P., Świerczewska-Gwiazda, A., Wiedemann, E.: Dissipative measure-valued solutions to the compressible Navier-Stokes system. Calc. Var. Partial Differ. Equ. 55, 141 (2016)

[6] Feireisl, E.: Dynamics of Viscous Compressible Fluids. Oxford University Press, Oxford (2003)

[7] Feireisl, E., Karper, T., Pokorný, M.: Mathematical Theory of Compressible Viscous Fluids: Analysis and Numerics. Springer, Berlin (2016)

[8] Feireisl, E., Jin, B.J., Novotný, A.: Relative entropies, suitable weak solutions, and weak-strong uniqueness for the compressible Navier-Stokes system. J. Math. Fluid Dyn. 14, 717-730 (2012)

[9] Feireisl, E., Lukáčová-Medvidová, M., Mizerová, H.: Convergence of finite volume schemes for the Euler equations via dissipative measure-valued solutions. Found. Comput. Math. 20, 923 (2019)

[10] Feireisl, E., Novotný, A.: Singular Limits in Thermodynamics of Viscous Fluids. Birkhäuser-Verlag, Basel (2009)

[11] Gwiazda, P., Świerczewska-Gwiazda, A., Wiedemann, E.: Weak-strong uniqueness for measure-valued solutions of some compressible fluid models. Nonlinearity 28, 3873-3890 (2015)

[12] Kato, T.: The Cauchy problem for quasi-linear symmetric hyperbolic systems. Arch. Rational Mech. Anal. 58, 181-205 (1975)

[13] Majda, A.: Compressible Fluid Flow and Systems of Conservation Laws in Several Space Variables, Applied Mathematical Sciences. Springer, New York (1984)

[14] Málek, J., Nečas, J., Rokyta, M., Ružička, M.: Weak and measure-valued solutions to evolutionary PDEs, Applied Mathematics and Mathematical Computation. Springer, Berlin (1996)

[15] Pan, R., Zhao, K.: The 3D compressible Euler equations with damping in a bounded domain. J. Differ. Equ. 246, 581-596 (2009)

[16] Schochet, S.: The compressible Euler equations in a bounded domain: existence of solutions and the incompressible limit. Comm. Math. Phys. 104, 49-75 (1986)

[17] Sideris, T.C., Thomases, B., Wang, D.: Long time behavior of solutions to the 3D compressible Euler equations with damping. Commun. Partial Differ. Equ. 28, 795-816 (2003)

[18] Sueur, F.: On the inviscid limit for the compressible Navier-Stokes system in an impermeable bounded domain. J. Math. Fluid Mech. 16, 163-178 (2014) 
Danica Basarić

Institute für Mathematik

Technische Universität Berlin

Straße des 17. Juni 136

10623 Berlin

Germany

e-mail: basaric@math.tu-berlin.de

Received: 6 March 2019.

Accepted: 3 October 2020. 